\title{
Late Eocene benthic foraminiferal fauna from clastic sequence of the Socka - Dobrna area and its chronostratigraphic importance (Slovenia)
}

\section{Poznoeocenska bentična foraminiferna favna iz klastičnega zaporedja med Socko in Dobrno in njen kronostratigrafski pomen}

\author{
Franc CIMERMAN ${ }^{1}$, Bogomir JELEN ${ }^{2}$ \& Dragomir SKABERNE ${ }^{2}$ \\ ${ }^{1}$ Paleontološki inštitut Ivana Rakovca ZRC-SAZU, Novi trg 2, SI -1000 Ljubljana \\ cimerman@zrc-sazu.si \\ ${ }^{2}$ Geološki zavod Slovenije, Dimičeva 14, SI -1109 Ljubljana \\ bogomir.jelen@geo-zs.si; dragomir.skaberne@geo-zs.si;
}

Key words: Benthic foraminifera, Upper Eocene, Priabonian, Socka beds, Socka, Dobrna, Slovenia

Ključne besede: bentične foraminifere, zgornji eocen, priabonij, soteške plasti, Socka, Dobrna, Slovenija

\begin{abstract}
We surveyed, sampled and described Late Eocene (Priabonian) benthic foraminiferal fauna from two sections in the type locality of the Socka beds, between Socka and Dobrna. We present a short history of research of these beds emphasising their dating, from the middle of $19^{\text {th }}$ century on. We describe the lithological characteristics and lithostratigraphy of the sections. In total 118 taxa of benthic foraminifers were identified, and the Priabonian age of the Soca beds established.
\end{abstract}

\section{Kratka vsebina}

$\mathrm{V}$ članku obravnavamo pozno eocensko (priabonijsko) bentično foraminiferno favno iz dveh profilov, ki smo ju posneli in vzorčevali na tipičnem ozemlju soteških plasti med Socko in Dobrno. V uvodu je predstavljena kratka zgodovina raziskovanj soteških plasti, predvsem problem njihove starosti, od sredine 19. stoletja do danes. Opisane so litološke značilnosti in litostratigrafija obeh profilov. Skupno je bilo določenih 118 taksonov bentičnih foraminifer in ugotovljena priabonijska starost soteških plasti.

\section{Introduction}

This paper contains only a portion of a broader Geological Survey of Slovenia project, Tertiary Tectofacies of the Southern Karavanke Mountains. Its goal was to describe and evaluate the stratigraphy of the
Southern Karavanke Tertiary, the type locality of the Socka beds between Socka and Dobrna (Fig. 1), and to compare it with the stratigraphy in other Slovenian Tertiary tectonostratigraphic units. The history section was written by Bogomir Jelen, the lithologic and lithostratigraphic description of 


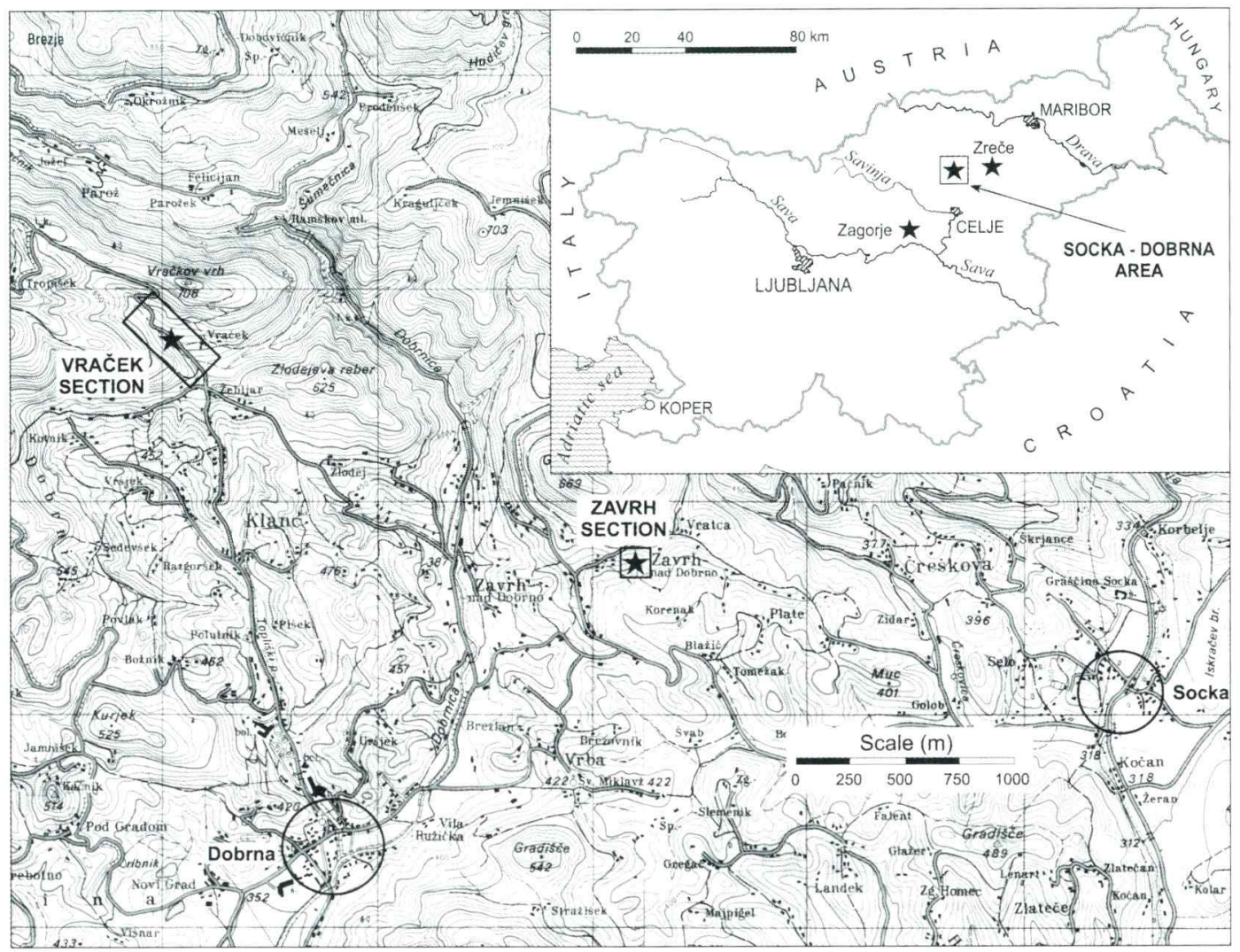

Fig. 1. Location map showing Socka-Dobrna area and detailed position of studied sections

sections by Dragomir Skaberne, and benthic foraminifers were described by Franc Cimerman.

\section{Historical development of the problem}

The problems associated with the stratigraphy of the Southern Karavanke Tertiary were recognized in the middle of the $19^{\text {th }}$ century because of interest in the fast-growing coal industry. Coal exposures are not rare in basal Tertiary beds in central and eastern Slovenia, at that time a part of the AustrianHungarian Empire. These were targeted by geologists from the just established State Geological Survey in Vienna. Initially they distinguished between higher grade Glanzkohle (German term for bituminous and subbituminous coal in English terminology), which occurred in the northern coal belt between the Drava and Savinja rivers, and the lower grade brown coal from the southern belt between the Savinja and Sava rivers.

Morlot (1849a) was the first to assign an Eocene age to the basal coal-bearing strata at Socka and Dobrna in the northern coal belt, and in 1850 the Miocene age of the southern, brown coal-bearing strata (note that the Oligocene Formation was established later, in 1854). Morlot's (1849a) statement was based on Unger's opinion (in Morlot, 1849b) of an Eocene age of the Socka macroflora. Influenced by the paleobotanist Buch (1851), Morlot in 1853 changed his mind, and accepted a Miocene age for the Socka flora. Thus he started the painful flip-flopping leaps from one age of the Socka beds to another and back, which lasted for a century and a half. Ettingshausen (1853) noted that the fossil macroflora from the Zagorje locality in the southern coal belt was like the Eocene flora with many Miocene species. 
Rolle (1857, 1858) first tackled "this hard problem" in his own words, after the introduction of the Oligocene as a new epoch between the Eocene and the Miocene Formations. He was not very successful, but he was probably the first researcher who used the term Socka beds (in German, Sotzka-Schichten; Rolle, 1858) for the basal Tertiary fresh water coal-bearing strata of the middle and northern coal belts. Namely, he introduced in the former north coal belt the middle coal belt, based on lithological differences and fossil content, but without evidence for age differentiation (Rolle, 1857). He (Rolle, 1858) assumed the Socka beds were Eocene and/or Oligocene in age. Ettingshausen (1858) published an article which revised Unger's flora and proposed a Late Eocene age of the Socka beds. Zollik ofer (1859) stated that the coal-bearing strata of the southern coal belt are Oligocene. In contrast, in his 1861/ 1862 work in which Zollikofer dealt with the area east of the Savinja river, he dismissed the Oligocene age and subdivided the coal-bearing strata in the southern coal belt between the Eocene to Oligocene and the earliest Neogene age.

Ettingshausen (1872, 1877, 1885) dealt with the fossil leaf assemblages from the southern coal belt in detail. The assemblage from the coal footwall indicated a tropical, or at least subtropical climate, whereas the assemblage from the hangingwall indicated it formed in the temperate one. It followed that the tropical/subtropical flora corresponded to the Eocene, and the temperate flora to the Aquitanian. Stur $(1864,1871)$ advanced the idea that Socka and Eibiswald (in Slovene: Ivnik) beds from the southern rim of the Graz basin are of the same age, i.e. of Early Neogene age. He (Stur, 1871) was the first to extend the term of Socka beds to the southern coal belt under the name Eibiswald and Socka beds. Bittner (1884) stated concisely that it was previously known that the Tertiary succession of the southern belt consists of two main rock units: the lower coal-bearing Socka beds unit, which is most probably of Oligocene age, and the upper unit which corresponds to the Neogene development of the Vienna basin in Austria. Hoernes (1876, 1892) temporarily solved the question of the age of the coal-bearing strata at the base of the Tertiary system. In 1892 he formulated the following conclusions (paraphrased): 1) Socka beds, fresh water coal-bearing strata of the northern and middle coal belt belong to a much older stage than the coal-bearing strata of the southern belt. In the northern coal belt they partly belong to the Cretaceous Formation, which is more or less proved, while the age of the other part of northern and middle coal belt is still problematic. With regard to the underlying Cretaceous coal-bearing strata of the northern coal belt, they are not considerably younger, but Eocene in age. 2) The Oligocene coalbearing strata in the southern coal belt have less in common with the Socka beds than they do with the Lower Miocene Eibiswald coal-bearing strata. Teller $(1889,1896$, 1898, 1907), expressed the opinion that Eocene deposits are completely missing. Tertiary deposits are divided into the Oligocene and the Miocene "lithostratigraphic" units. The coal-bearing strata comprise the youngest unit of the Oligocene succession in the northern, middle and southern coal belts.

Petrascheck (1926/1929) summarized existing stratigraphic ideas. He considered all coal-bearing strata at the base of the Tertiary System to be Socka beds and accepted their Oligocene age. Papp (1954, 1955) inferred the Chattian (Upper Oligocene) age from the Miogypsina (Miogypsinoides) cf. formosensis Jabe \& Hanzawa and Lepidocyclina (Nephrolepidina) tournoueri (Lemoine \& Douvillé) found in the conformable marine marly clay, overlying the Socka beds of the southern coal-belt. $\mathrm{Ku}$ ščr (1955) proposed the idea that the Chattian rocks were missing in the southern coal belt. Szöts (1956) reasoned that the Chattian stage was the equivalent of the Aquitanian stage, therefore, according to him, the Socka beds were of the Aquitanian, i.e. of Early Miocene age, with Oligocene layers completely missing. Rijavec (1958) followed Papp's view. Majzon (1958) argued for a Lattorfian (Lower Oligocene) age of the Socka beds. Thenius (1959) was convinced that Anthracotherium illyricum Teller and Miogypsina (Miogypsinoides) cf. formosensis Jabe \& Hanzawa indicated the (late) Stampian (late Chattian) age of the Socka beds in the southern coal belt.

Ku ščr's (1967) stratigraphic positional relationship scheme was based on the Rupe- 
lian (Middle Oligocene) age of the Socka beds and the conformable overlying marine marly clay in the southern and middle coal belts. Their age was determined by the characteristic benthic microforaminiferal taxa accompanying Tritaxia szaboi (Hantken) from the marine marly clay which exhibited a close similarity with the Middle Oligocene (at that time Rupelian) microforaminiferal fauna of Hungary and Transylvania (NW Rumania). Then, in 1975, Papp came forth with new taxonomic results: his Miogypsina (Miogypsinoides) formosensis Jabe \& Hanzawa and Miogypsina (Miogypsinoides) bantamensis Tan Sin Hok from the marine marly clay overlying Socka beds in the southern coal belt indicated a late Early Egerian (= late Chattian, Late Oligocene) age. From the map of Mioč \& Žnidarčič (1977), one can conclude that at the Socka site, the coal-bearing strata are Helvetian s.l. in age - equivalent to Ottnangian/Karpatian. Cimerman (1979) kept the Rupelian age for the "Socka" beds in the southern coal belt. He (ibid.) stated that the use of term Socka beds in the southern coal belt is not justified and proposed to write Socka in quotation marks - "Socka" beds. The same year saw revival of the idea of Eocene age deposits in central Slovenia. Mikuž (1979) advanced the thesis that the Socka beds of the western Southern Karavanke Mts. (middle coal belt) were not Rupelian, but Middle Eocene in age.

Drobne et al. (1979) published a Late Eocene age of a very small limestone patch in the eastern Southern Karavanke Mts. Mi o č (1978) distinguished between the Middle Oligocene and the Helvetian s. 1. Socka beds in the Socka-Dobrna type area. Buser (1979) and Premru (1983) retained the Middle Oligocene (Rupelian) age of the southern coal belt Socka beds, while Buser allowed their Eocene age in the middle coal belt, though not naming them as such. Aničić \& Juriša (1985a) avoided the term Socka beds. In the southern coal belt Aničić \& Juriša (1985a, b) recognized both, the Middle Oligocene (Rupelian) and the Late Oligocene (Chattian) coal-bearing strata. In the middle coal belt, Aničić \& Juriša (1985a, b) assigned the Helvetian s.l. age to the basal coal-bearing strata, remarking that an older age was possible. B ald i (1984) correlated the Socka beds with the nano- plakton zone NP 23, (Rupelian). Drobne et al. (1985) correlated the Socka beds of the western Southern Karavanke Mts. (middle coal belt) with the late Middle Eocene, and the Socka beds of the southern coal belt with the Early Oligocene NP 21/22.

Hamrla (1987) presented a detailed stratigraphy of the Zreče area in the northern coal belt. He distinguished between the Late Cretaceous, older Paleogene and Helvetian s.l. coal-bearing beds. However, Brezigar (in Brezigar et al. 1988) was the first to speculate about the Eocene age of the Socka beds in the whole Southern Karavanke geotectonic unit, and thought that the Tertiary stratigraphy might be different within the Southern Karavanke and Savinja Alps geotectonic units. Royden \& Baldi (1988) once again correlated the Socka beds of the middle and southern coal belt with the NP 23 zone. In the same year Mihajlović \& Jungwirth (1988) also placed the Socka beds of the southern coal belt into the NP 23 zone, as did P a v šič and Jelen (in Jelen et al., 1990). Csontos et al. (1992) considered the lithothamnium limestone at the base of the Tertiary succession in the southern coal belt to be Late Eocene, followed by a gap between it and the overlying "Socka" beds of the NP 23 zone. In 1992, our working group, dedicated to solving the problem of this high stratigraphic inconsistency, published the first note on its work (Jelen et al., 1992).

The above summary shows how confusing and instable the position of the basal Tertiary coal-bearing strata was. Since each change in their age attribution resulted into changes in age upsection, and accordingly a revision of their stratigraphical relationships, we can understand how critical the drastic age inconsistency of the basal coalbearing strata was.

The problem was to find a solution to the issue or, how to get the key to go to the root of the problem that had been baffling so many past workers.

The first palynological inspection of the coal-bearing strata at the base of the Tertiary successions showed great differences in the composition of palynological assemblages from the northern, the middle, and the southern coal belts (Jelen, unpublished). After palynological analysis we applied a structural-stratigraphic modelling to 
establish an as much as possible flawless lithochronology of the middle coal belt, in which the Socka-Dobrna type area is situated. Luckily, in the type area the lithochronology could be satisfactorily established. Sampling was first performed in successions where the lithochronology was considered to be well established. Foraminiferal samples were collected from one metre long trenches in 1-metre intervals, if outcrop conditions were favourable, or at each metre, if considered necessary. In addition, we developed a new laboratory preparation method that allowed us to recover highly concentrated foraminifers (Jelen, unpublished).

\section{Lithology and lithostratigraphy in the Vraček section area}

The composite Vraček section (Vr/I and $\mathrm{Vr} / \mathrm{II}$ ) is situated north of Dobrna, south of Vračkov vrh $(708 \mathrm{~m})$, along the way from the Žebljar farm north of the Klanec village toward the Trojnšek farm (Figs. 1, 2a). This area is more structuraly complex than was expected at the beginning of field work.

Tectonically disturbed limestones and pyroclastic rocks of Triassic age and older Eocene limestones are overlain by Upper Priabonian (J el en et al., 2000), mostly clastic sedimentary rocks. The contact is partly tectonized. Eocene beds were folded together with basement rocks. All units are unconformably overlain by Miocene coarse-grained rocks with remains of ostrean valves of the Klanec beds and sandy marly rocks of the Dobrna marl.

Eocene beds are slightly bent into a syncline. The area is cut by several Dinaric (NW - SE), subvertical, sinistral faults into narrow, 20 to $60 \mathrm{~m}$ wide bands or wedges. Within the bands the Eocene marly beds are compressed, crushed and fissile. Fissility is steep and chiefly fault-parallel. In non-fissil parts, the rocks are predominantly massive, in places faintly laminated. The section comprises somewhat more than $200 \mathrm{~m}$ of the Eocene beds.

In the first, shorter segment of section, which is separated from the second main segment by a fault, Eocene rocks occur on tectonically disturbed green volcaniclastic rocks (tuffs, tuffites with thin intercalations of dark grey biomicritic limestones) of presumably Middle Triassic age.

The Eocene succession starts with a one meter thick, prevailingly light to medium grey fine-crystalline dolomite (unit $\mathbf{1}$ on Fig. $2 \mathrm{a})$. The rock contains lenses, laminae and thin layers of xylitic coal, $2 \mathrm{~mm}$ to $5 \mathrm{~cm}$ thick in its lower half. In the upper part traces of plant root systems can be seen as well as limonitized, originally pyritic concretions.

The dolomitic bed is followed by a $1.7 \mathrm{~m}$ thick clayey coal interval (unit 2 on Fig. 2a). It consists of 5 to $30 \mathrm{~cm}$ thick layers of carbargillitic, detritic and xylitic coal and two 2 to $5 \mathrm{~cm}$ thick predominantly clayey beds with a lower content of organic, coaly components

Above the coaly interval follows a dark yellowish brown, somewhat weathered, marly silty shale of weakly expressed parallel lamination (unit $\mathbf{3}$ on Fig. 2a) The rock can be compared to weathered yellowish grey marly silty shale at the beginning of the second, main part of the section. That rock is $14 \mathrm{~m}$ thick, it overlies the tectonized limestone with numerous calcitic veins, and is probably Triassic in age.

In the second part of the section, the yellow grey marly silty shale passes into brownish grey to dark brownish massive silty marlstone (unit 4 on Fig. 2a) approximately $20 \mathrm{~m}$ thick. It contains numerous ostracods and occasional bivalve and gastropod shells.

The dark brownish massive silty marlstone passes into yellowish grey, somewhat weathered marly silty shale (unit $\mathbf{5}$ on Fig. 2a) comprising a thickness of $7 \mathrm{~m}$, up to the contact with a limestone lens.

The limestone lens (unit $\mathbf{6}$ on Fig. 2a) is $5.2 \mathrm{~m}$ thick and can be genetically divided in two parts. The lower part, $4.2 \mathrm{~m}$ thick, bordering along a sharp contact on the underlying yellowish grey marly silty shale, was macroscopically subdivided into three sequences. They start with up to $15 \mathrm{~cm}$ thick layers of calcirudite with angular clasts of intramicrite and biointramicrite up to $4 \mathrm{~cm}$ in size. On the lower side of these layers and within overlying macroscopically homogeneous, 0.7 to $2.4 \mathrm{~m}$ thick intervals of biomicritic limestone stylolites parallel to bedding are visible. In the upper part the lens is terminated with a $1.2 \mathrm{~m}$ thick normally graded carbonate turbiditic sequence. 

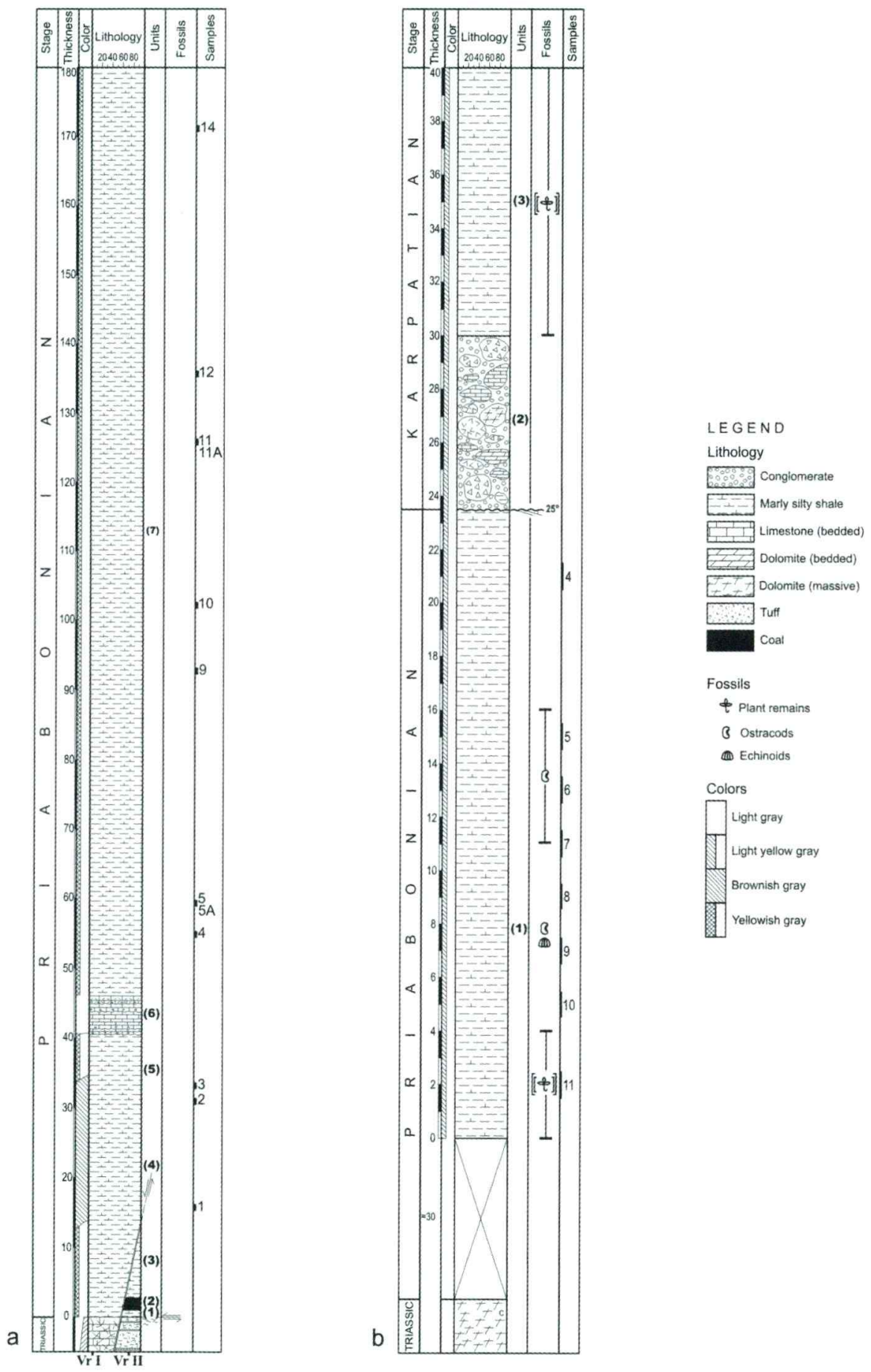

Fig. 2. Stratigraphic columns of:

a) Vraček section (Vr I and Vr II)

b) Zavrh section (Zv II). Karpatian part was also determined by foraminifers but this is not a topic of this article 
Above the limestone lens lies a medium to light grey, partly weathered yellowish grey, tectonically disturbed marly silty shale (unit 7 on Fig. 2a). Macrofossils are rare. In places rare plant remains can be found. In the section above the limestone lens more than 150 $\mathrm{m}$ of real thickness of these rocks are comprised.

The lithostratigraphically highest part of marly rocks (of lithostratigraphic unit $\mathbf{7}$ ) in the Vraček section is exposed in the Zavrh area, where it was covered with the Zavrh (Zv) section.

\section{Lithology and lithostratigraphy in the Zavrh section area}

The Zavrh ( $\mathrm{Zv})$ section is situated between Zavrh and Čreškova in the area of the Vratca settlement (Figs. 1, 2b). The lower part of Tertiary beds to pre-Tertiary rocks is covered. Along the cartway leading to Čreškova, however, they are abundantly exposed. Approximately parallel to the section a fault passes in the NW-SE direction. The horizontal component of the dextral displacement along the fault amounts to about $27 \mathrm{~m}$.

The contact between the pre-Tertiary basement and Tertiary rocks is tectonical. In the basement a crystalline dolomite (dolosparite) occurs, on which erosional remains of red, probably weathered muddy rocks occur higher in the slope. The Tertiary sequence starts with a light grey silty clay. The thickness of the covered part of Tertiary rocks is an estimated $30 \mathrm{~m}$. In rare outcrops, a grey massive to slightly laminated silty marlstone with rare plant remains occurs. The rock is brownish weathered and exhibits spheroidal parting. In the lower part occur rare, up to $0.1 \mathrm{~m}$ thick layers of biodolomicrite with terrigenous admixture.

In the area of the carefully logged Zavrh $(\mathrm{Zv} / \mathrm{II})$ section there is a weathered light yelow grey silty marlstone with poorly expressed lamination in its lower part (unit $\mathbf{1}$ on Fig. 2b). Additionally, there are plant remains among the fossils, and in the middle part ostracods and rare echinoderm fragments. We found no fossils in the upper portion of $\mathrm{Zv} / \mathrm{II}$.

Following an angular discordance $\left(25^{\circ}\right)$, a $6.5 \mathrm{~m}$ thick lenticular body of blocky conglomerate (unit $\mathbf{2}$ on Fig. 2b) overlies the silty marlstone. The blocky conglomerate is cut off in the SW by the aforementioned fault that passes parallel to the section. Toward the NE the conglomerate thins and becomes finer grained. It contains numerous fossil fragments: of red algae (lithothamnia), bivalves, bryozoans, echinoderms and of other organisms. The blocky conglomerate consists of approximately $75 \%$ blocks and pebbles and $25 \%$ of muddy matrix. Blocks and pebbles range in size from 1 to $100 \mathrm{~cm}$, on an average $20 \mathrm{~cm}$, and are very poorly sorted. The size distribution of pebbles and blocks is bimodal. The first mode is in the range from 1 to $20 \mathrm{~cm}$, and the second one in the 30 to $100 \mathrm{~cm}$ range. Nineteen blocks, 20 $\mathrm{cm}$ or more in size, and 4 smaller pebbles were examined microscopically. In these we recognized 11 lithofacies, represented in 1 to 4 blocks or pebbles: dolomitic breccia with limonitized dolomicritic cement, dolomicrosparite with brecciated structure, crystalline dolosparite, dolomitized biomicrite, altered crystallolithic (andesitic) volcaniclastic rock, calcareous dolomite breccia, calcitic micaceous quartzy very fine-grained sandstone, partly recrystallized volcanic glass with microliths, recrystallized intrabiomicrite, intrabiomicrite with admixture of terrigenous components, and biopeldolomicrite. Blocks and pebbles are of Triassic to inclusively Eocene age, and are derived in part directly from the underlying rocks.

The blocky conglomerate is followed at the end of the section by monotonous parallelly laminated, slightly micaceous marly silty shale (unit 3 on Fig. 2 b) with rare up to $1 \mathrm{~mm}$ thick lenses of very fine-grained sandstone. Along with rare plant remains, visibile with naked eye, there is also a foraminiferal fauna.

\section{Benthic foraminiferal fauna from the Socka beds}

Eocene benthic foraminifers from Vraček II (Vr/II-) and Zavrh II (Zv/II-) sections were studied. In the two sections a total of 118 taxa were determined, belonging to 85 genera and 53 families.

\section{Vraček II}

From the VRAČEK II section (Fig. 2a) 12 samples (Vr/II-001, 002, 003, 004, 005, 005A, 
$009,010,011,011 \mathrm{~A}, 012,014)$ were analyzed. In the section occur 107 taxa, out of them 49 only here. The highest number of taxa, 53, were found in sample Vr-005A, 39 in sample $\mathrm{Vr}-005$, and in the remaining samples from 2 to 29 taxa.

\section{Zavrh II}

From the ZAVRH II section (Fig. 2b) 8 samples were analyzed, (Zv/II-011, 010, 009, 008, 007, 006, 005, 004, samples in sections are numbered in stratigraphic order, from the bottom upwards). In the section occur 75 taxa, of them 12 only here. The maximum taxa number was found in $\mathrm{Zv} / \mathrm{II}-6,37$, and the smallest in the uppermost collected sample Zv/II-004, only 6; otherwise the number of taxa varies in a sample between 21 and 37 .

\section{Ecological interpretation of foraminiferal fauna}

In samples Vr/II-001, 002 no foraminifers are present, only ostracods. The first foraminifers appear in sample $\mathrm{Vr} / \mathrm{II}-003$ as individual hauerinids: Quinqueloculina sp. 3 and Pyrgo subsphaerica (d'Orbigny). This part of the Vraček II section represents the passage from fresh water or brackish environment into a marine setting. Foraminifers become more numerous in sample Vr/II-004, which is entirely marine. The Zv II section is wholly marine. In samples Vr/II-004, 005, 005A the following large foraminiferal genera occur: Heterostegina sp., Operculina cf. gomezi (two juvenile forms), Discocyclina trabayensis vicenzensis (this species was determined by Vlasta Ćosović), Asterocyclina sp., Eoannularia eocenica, and the large miliolid Borelis vonderschmitti. The species Calcarina lecalvezae was established in both sections (Vr-014, Zv-009). These are shallow marine species, and are, according to their ecological characteristics, in contradiction with the remaining foraminiferal fauna in the same samples. Therefore we presume that the fauna was transported, washed out from shallower areas into the deeper sea. In the Vraček II section appears in marl a limestone lens with discocyclinas (the foraminiferal fauna has not yet been determined and interpreted). In Fig. 2a the lens is marked with number 6 . Also at the
Povlak farm, about $1 \mathrm{~km}$ NW of Dobrna, an Eocene limestone with nummulites, discocyclinas, Borelis, and corals is exposed. The limestone at Povlak and the limestone lens at the Vraček II section may represent remains of sediments of the shallow sea from which the large foraminifers were redeposited. For some more frequent genera, Murray (1991, 288-290 and 323-326) reports ecological data from recent environments. Among them appear 20 genera that are identical to genera from our sections. For each of these genera the habitat extends from the shelf (either internal or external) to the bathyal. The shelf is indicated also by the miliolids (Quinqueloculina). The upper slope (200-2000 m) is indicated by genera Ammobaculites, Bathysiphon, Karreriella, Repmanina, Textularia, Bulimina, Globocassidulina, Hoeglundina, Pullenia, Reussella, Trifarina and Uvigerina. The genus Laticarinina that was found in both sections is an indicator of even greater bathyal depths. However, the observation of a relatively small number of planktonic forms is consistent with deposition of the sediments in the bathyal domain, but rather on the exernal shelf with a passage to the bathyal. According to the bathymetric interpretation of sediments at Biarritz (Gruenig \& Herb, 1984) they were deposited in the realm extending from neritic to bathyal.

\section{Comparison with Late Eocene faunas in Europe}

By comparisons of the occurrence of the species in Vraček II and Zavrh II sections with those in other European localities of the Eocene (Possagno in north Italy, Buda marl in Hungary and Upper Eocene beds with Tritaxia szaboi in Hungary and Biarritz in France) we want to demonstrate the degree of similarity of our fauna with them.

In north Italy, B rag a et al. (1975) established in Paleocene, and Lower and Middle Eocene at Possagno 99 benthic species, of which $10(=8 \%)$ are also our species. From the Upper Eocene 126 benthic species were determined. Among these $13 \quad(=11 \%)$ are found in our sections.

In the Buda marl Sztràkos (1982) established 471 forms of foraminifers, out of these 416 were determined to the species or subspecies level. $42(=35 \%)$ of our species 
are identical to those. From the Bartonian and Priabonian beds with Tritaxia szaboi, Sztràkos (1987) determined 429 species. Of these, $45(=38 \%)$ are also our species.

The fauna from the Biarritz locality was investigated by Halkyard (1919), Gruenig \& Herb (1984), Gruenig (1985) and Sztràkos (In: Mathelin \& Sztràkos, 1993). Halkyard (1919) established the presence of 345 foraminiferal species. Gruenig (1985) determined 87 species. By far the most complete review was performed by Sztràkos (In: Mathelin \& Sztràkos, 1993) who recorded 557 species. With the species from Biarritz $77(=65 \%)$ species are identical, or two thirds of species from the Vraček II and Zavrh II sections are found in Biarritz. Outstanding among them is Marginulina globosa Halkyard. It was previously known only from Biarritz, and Halkyard's specimen was the only one of this species until Sztràkos found additional three individuals in the Aquitanian basin in beds of the zone with Turborotalia cerroazulensis s. 1 . (Middle Priabonian) (Sztràkos, 1993, 44. In: Mathelin \& Sztràkos). The species is obviously very rare. Our find is from the third and easternmost locality. The species is interesting and important as an indicator of the connection of our Late Eocene sea toward the west.

\section{Dating of the Socka beds}

For dating the species of benthic foraminifers that are restricted to Eocene and that do not survive the Eocene-Oligocene boundary are critical. There are 22 such species (Heterostegina sp. does not belong to them) in our two sections:

\section{Pavonitina biarritzensis}

Dorothia fallax

Karrerotextularia olianensis

Sigmoilopsis bartoniensis

Borelis vonderschmitti

Reticulopalmula arborescens

Marginulina globosa

Spirolingulina acutimargo

Ceratocancris crassa

Lamarckina cristellaroides

Sporobulimina eocaena

Sagrinopsis aspera

Tubulogenerina tubulifera
Uvigerina chirana

Schlosserina asterites

Laticarinina altocamerata

Halkyardia minima

Melonis halkyardi

Eoannularia eocenica

Calcarina lecalvezae

Operculina cf. gomezi

Discocyclina trabayensis vicenzensis

Serra-Kiel et al. (1998) proposed the biozoning of the Paleocene and Eocene on the basis of shallow marine benthic foraminifers in $20 \mathrm{SB}$ (shallow benthic) biozones. The last two biozones, SB 19 and SB 20, comprise the Priabonian (upper part of NP 18 to lowermost part of NP 21 or upper part of P 15 to P 17. After Serra-Kiel et al. 1998) Several species extend vertically through biozones, in our sections. These are:

- Borelis vonderschmitti which comprises the top part of SB 18, the whole SB 19 and lower part of SB 20

- Operculina gomezi covers the upper part of SB 18, entire SB 19 and entire SB20

- Halkyardia minima comprises the zones SB 15 to the end of SB 20

- Discocyclina trabajensis vicenzensis comprises SB 20

- Calcarina lecalvezae comprises the zones SB 17 and SB 18.

The authors of Calcarina lecalvezae, Boulanger \& Poignant $(1971,96)$, report the for Upper Eocene for its range, but they also found it in the Upper Lutetian. Heterostegina is also stratigraphically important and is represented by a few individuals in the Vraček II section; it does not appear before the Priabonian (Hotting er, 1977, 142; Loeblich \& Tappan, 1987, 684).

The most data for detailed dating could be obtained from planktic foraminifers. However, they are rare relative to benthic foraminifers. From samples Vr/II-005, 010, 011, 011A, 014 and Zv/II-009 Monique Toumarkine determined 15 planktic species belonging to four genera and three families. In a written communication she qualified them as species without great stratigraphic value, as for example Catapsidrax dissimilis (Cushman \& Bermudez), Globorotalia suteri Bolli, the range of which extends from the Eocene into the Miocene. The other ones are globigerinas from the Eocene-Oligocene boundary. Only speciesTurborotalia cerro- 
azulensis cerroazulensis (Cole) indicates a Late Eocene age. Foraminifers are not very well preserved, they are often deformed and their apertures filled with sediment.

The benthic and planktic foraminifers permit us to conclude that the beds in the Vraček II and Zavrh II sections are Priabonian in age, which also confirms the Priabonian age of the Socka beds.

\section{Systematics}

Order FORAMINIFERIDA Eichwald, 1830 Family: Bathysiphonidae Avnimelech, 1952

\section{Bathysiphon taurinensis Sacco, 1893}

Pl. 1, Fig. 1

1893 Bathysiphon taurinensis - Sacco, p. 168, pl. 11, fig. 2 (Fide Ellis \& Messina 1940)

1975 Bathysiphon taurinense Sacco-Popescu, p. 32 , pl. 1, fig. 2

Locality: Vr-005, Vr-005A, Zv-008, Zv009, Zv-011

Family: Ammodiscidae Reuss, 1862

\section{Repmanina charoides (Jones \& Parker), 1860}

1860 Trochammina squamata Jones \& Parker var. charoides - Jones \& Parker, p. 304 (Fide Ellis \& Messina 1940)

1982 Glomospira charoides (Jones \& Parker) Foraminiferi padani, pl. 1, fig. 3

1975 Glomospira charoides (Jones \& Parker) Braga et. al., p. 102

Locality: Zv-010

Family: Lituolidae de Blaiville, 1827

\section{Ammobaculites agglutinans (d'Orbigny),} 1846

Pl. 1, Figs. 2-3

1846 Spirolina agglutinans - d'Orbigny, p. 137, pl. 7, figs. 10-12

1985 Ammobaculites agglutinans (d'Orbigny) Papp \& Schmid, p. 196, pl. 45, figs. 6-9

1985 Haplophragmoides - Ammobaculites sp. Grünig, p. 255, pl. 1, figs. 10-12

1993 Ammobaculites agglutinans (d'Orbigny) Sztràkos (In: Mathelin \& Sztràkos), p. 73, pl. 1, fig. 15

Locality: Vr-012

Family: Spiroplectamminidae Cushman, 1927
Spiroplectinella dalmatina (de Witt

Puyt), 1941

Pl. 1, Fig. 4

1941 Textularia dalmatina - de Witt Puyt, p. 45, pl. 1, figs. 4-5

1975 Spiroplectammina dalmatina (de Witt Puyt) - Braga et al., p. 102

1985 Spiroplectammina dalmatina (de Witt Puyt) - Grünig, p. 256, pl. 2, figs. 1-4

Locality: Vr-010, Zv-008, Zv-009

Vulvulina haeringensis (Gümbel), 1868

Pl. 1, Fig. 6

1868 Venilina haeringensis - Gümbel, p. 71, pl. 2, fig. 84

1975 Vulvulina haeringensis (Gümbel) - Braga et al., p 91, pl. 1, figs. 4, 23; p. 102

1985 Vulvulina haeringensis (Gümbel) - Grünig, p. 256, pl. 2, figs. 10-12

1993 Vulvulina haeringensis (Gümbel) - Sztràkos (In: Mathelin \& Sztràkos), p. 73, pl. 2, fig. 12

Locality: Vr-004, Vr-005, Vr-005A, Vr-

009, Vr-011, Vr-012, Zv-006, Zv-008

Family: Pavonitinidae Loeblich \& Tappan, 1961

\section{Pavonitina biarritzensis Sztràkos, 1987}

Pl. 1, Figs. 7-8

1987b Pavonitina biarritzensis - Sztràkos, p. 129 , pl. 1 , figs. $1-3$, pl. 3 , figs. $1-3$, textfig. 1

1993 Pavonitina biarritzensis Sztràkos - Sztràkos (In: Mathelin \& Sztràkos), p. 73, pl. 24, fig. 5

Locality: Vr-005A, Zv-005, Zv-006, Zv-

008, Zv-010, Zv-011

Family: Prolixoplectidae Loeblich \&

Tappan, 1985

\section{Plectina dalmatina (Schubert), 1811}

Pl. 1, Figs. 9-10

1911 Gaudryina dalmatina Schubert - Liebus, p. 75, pl. 3, fig. 5

1919 Clavulina gaudryinoises Halkyard non Fornasini - Halkyard, p. 46, pl. 3, figs. 1-3

1937a Plectina dalmatina (Schubert) - Cushman, p. 107, pl. 12, fig. 8

1985 Plectina dalmatina (Schubert) - Grünig, p. 158, pl. 3, figs. 1-6

Locality: Vr-004, Vr-005, Vr-009, Vr-010, Vr-012, Zv-006, Zv-007, Zv-009, Zv-010, $\mathrm{Zv}-011$ 
PLATE 1

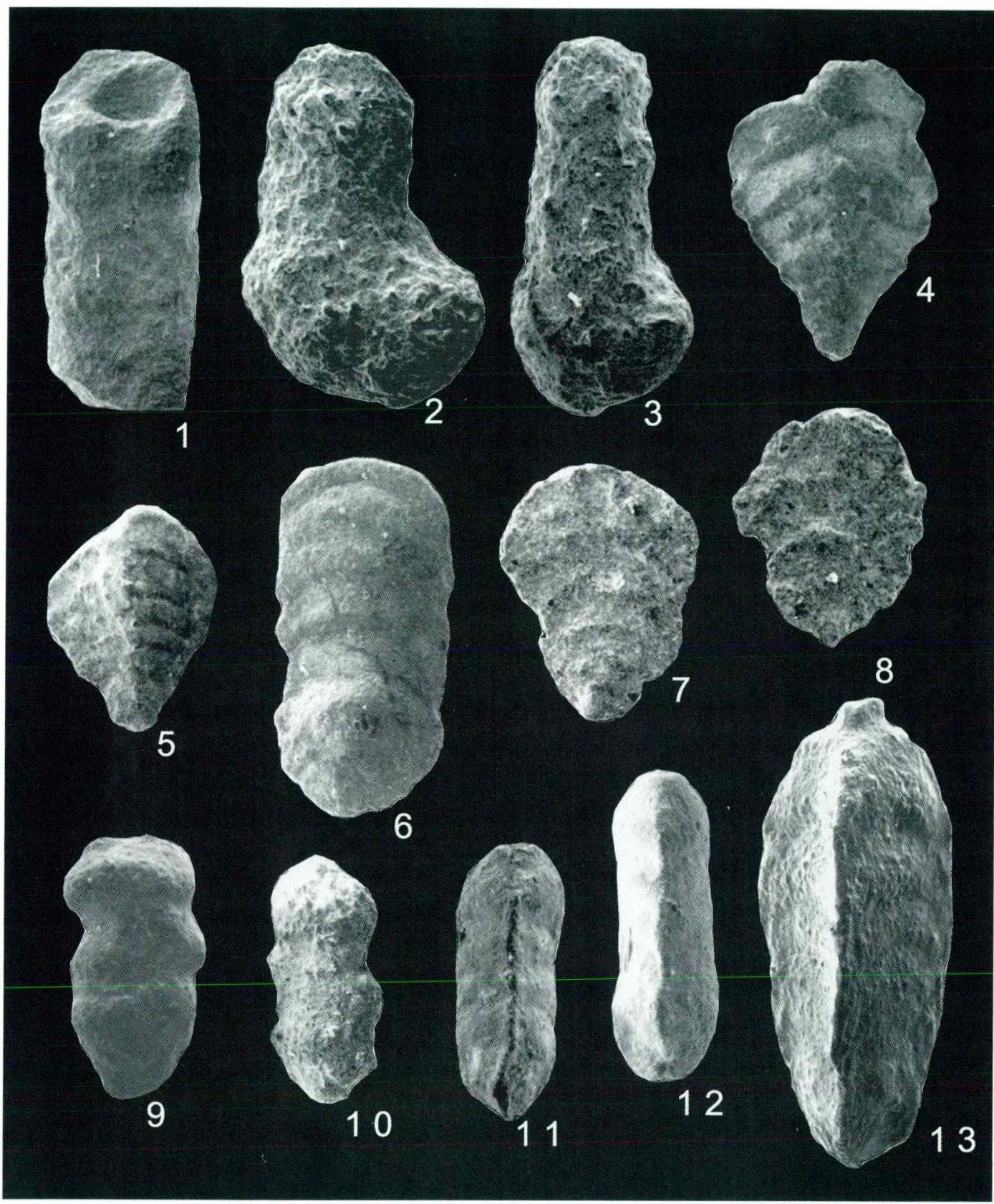

1 Bathisyphon taurinensis Sacco, Vr-009, x28. 2-3 Ammobaculites agglutinans (d'Orbigny). 2 Vr-012, x40. 3 Vr-012, x40. 4 Spiroplectinella dalmatina (de Witt Puyt), Vr-009, x60. 5 Spiroplectinella pectinata (Hantken), Vr-010, x40. 6 Vulvulina haeringensis (Gümbel), Vr-009, x40. 7-8 Pavonitina biarritzensis Sztràkos. 7 Zv/II-006, x40. 8 Zv/II-006, x40. 9-10 Plectina dalmatina (Schubert). 9 Vr-009, x40, 10 Vr009, x40. 11-12 Tritaxia kruhelensis (Woicik). 11 Vr-012, x40. 12 Vr-012, x40. 13 Tritaxia szaboi (Hantken), Vr-009, x40. 
Family: Tritaxiidae Plotnikova, 1979

\section{Tritaxia kruhelensis (Woicik), 1903}

Pl. 1, Figs. 11-12

1903 Clavulina szaboi var. kruhelensis - Woicik, p. 498, pl. 6, fig. 21 (Fide Ellis \& Messina 1940)

1956 Clavulinoides kruhelensis (Woicik) - Hagn, p. 117 , pl. 10 , figs. $2-4$, 8-9

1993 Clavulinoides kruhelensis (Woicik) Sztràkos (In: Mathelin \& Sztràkos), p. 73, pl. 3, fig. 19

Locality: Vr-012, Zv-004

\section{Tritaxia szaboi (Hantken), 1868}

Pl. 1, Fig. 13

1868 Clavulina Szaboi - Hantken, p. 83, pl. 1, figs. $4,6,7$

1868 Rhabdogonium Szaboi - Hantken, p. 90, pl. 1, fig. 18

1956 Clavulinoides Szaboi (Hantken) - Hagn, p. 116, pl. 10, fig. 1

1975 Tritaxia szaboi (Hantken) - Braga et al. p. 103, pl. 4, figs. 1-2

1993 Clavulinoides szaboi (Hantken) - Sztràkos (In: Mathelin \& Sztràkos), p. 73, pl. 4, fig. 1 ; pl. 25 , fig. 5

Locality: Vr-004, Vr-005A, Zv-005, Zv-

006, Zv-008, Zv-010, Zv-011

Family: Eggerellidae Cushman, 1937

\section{Dorothia fallax Hagn, 1956}

Pl. 2, Figs. 1-2

1956 Dorothia fallax - Hagn, p. 119, pl. 9, figs. 18-19; pl. 18, fig. 10

1975 Dorothia fallax Hagn - Braga et al., p. 92, pl. 1, fig. 25

1993 Dorothia fallax Hagn - Sztràkos (In: Mathelin \& Sztràkos), p. 39, pl. 24, fig. 8

Locality: Zv-010, Zv-011

\section{Karreriella halkyardi Cushman, 1936}

Pl. 2, figs. 3-4

1936 Karreriella halkyardi - Cushman, p. 36, pl. 5, fig. 16

1985 Karreriella halkyardi Cushman - Grünig, p. 258, pl. 3, figs. 21-22

1993 Karreriella halkyardi Cushman - Sztràkos (In: Mathelin \& Sztràkos), p. 39, pl. 24, fig. 9

Locality: Vr-004, Vr-005, Vr-005A, Vr-

010, Zv-004, Zv-006, Zv-008, Zv-009, Zv010

Martinottiella $s p$.

Pl. 2, Fig. 5
Locality: Vr-004

Family: Textulariidae Ehrenberg, 1838

Semivulvulina pectinata (Hantken), 1875

P1. 1, Fig. 5

1875 Vulvulina pectinata - Hantken, p. 68, pl. 7, fig. 10

1975 Vulvulina aff. pectinata Hantken - Braga et al. p. 102 . pl. 4 , fig. 3

1985 Spiroplectammina aff. pectinata (Hantken) - Grünig, p. 256, pl. 2, figs. 5-8

1993 "Semivulvulina" cf. pectinata (Reuss) Sztràkos (In: Mathelin \& Sztràkos), p. 40, pl. 2, fig. 11

Locality: Vr-005A, Vr-009, Vr-010, Vr-

011, Vr-012, Vr-014, Zv-005, Zv-006, Zv-

$008, \mathrm{Zv}-010$

Textularia adalta Cushman, 1926

Pl. 2, Fig. 6

1926 Textularia adalta - Cushman, p. 29, pl. 4, fig. 2 (Fide Ellis \& Messina 1940)

1950 Textularia adalta Cushman - Ruiz de Gaona \& Colom, p. 416, figs. 5/22-26

1985 Textularia adalta Cushman-Grünig, p. 257, pl. 3, figs. 16-17

1993 Textularia adalta Cushman - Sztràkos (In: Mathelin \& Sztràkos), p. 73, pl. 3, fig. 9

Locality: Vr-005, Vr-005A, Vr-009, Zv-010

Karrerotextularia olianensis (Ruiz de

Gaona \& Colom), 1950

Pl. 2, Figs. 7-8

1950 Siphotextularia olianensis - Ruiz de Gaona \& Colom, p. 413, figs. 16/1-9

1985 Siphotextularia concava (Karrer) - Grünig, p. 257, pl. 2, figs. 18-19

1991 Siphotextularia olianensis Ruiz de Gaona \& Colom - Barbin \& Keller-Grünig, p. 241, pl. 1 , figs. 3-4

1993 Karrerotextularia olianensis (Ruiz de Gaona \& Colom) - Sztràkos (In: Mathelin \& Sztràkos), p. 40, pl. 3, figs. 17-18; pl. 25, figs. $3-4$

Locality: Vr-009

Family: Pseudogaudryinidae Loeblich \&

Tappan, 1985

\section{Pseudogaudryina textilaroides (Hantken),}

1875

Pl. 2, Fig. 9

1875 Gaudryina textilaroides - Hantken, p. 15, pl. 1 , fig. 6 
PLATE 2

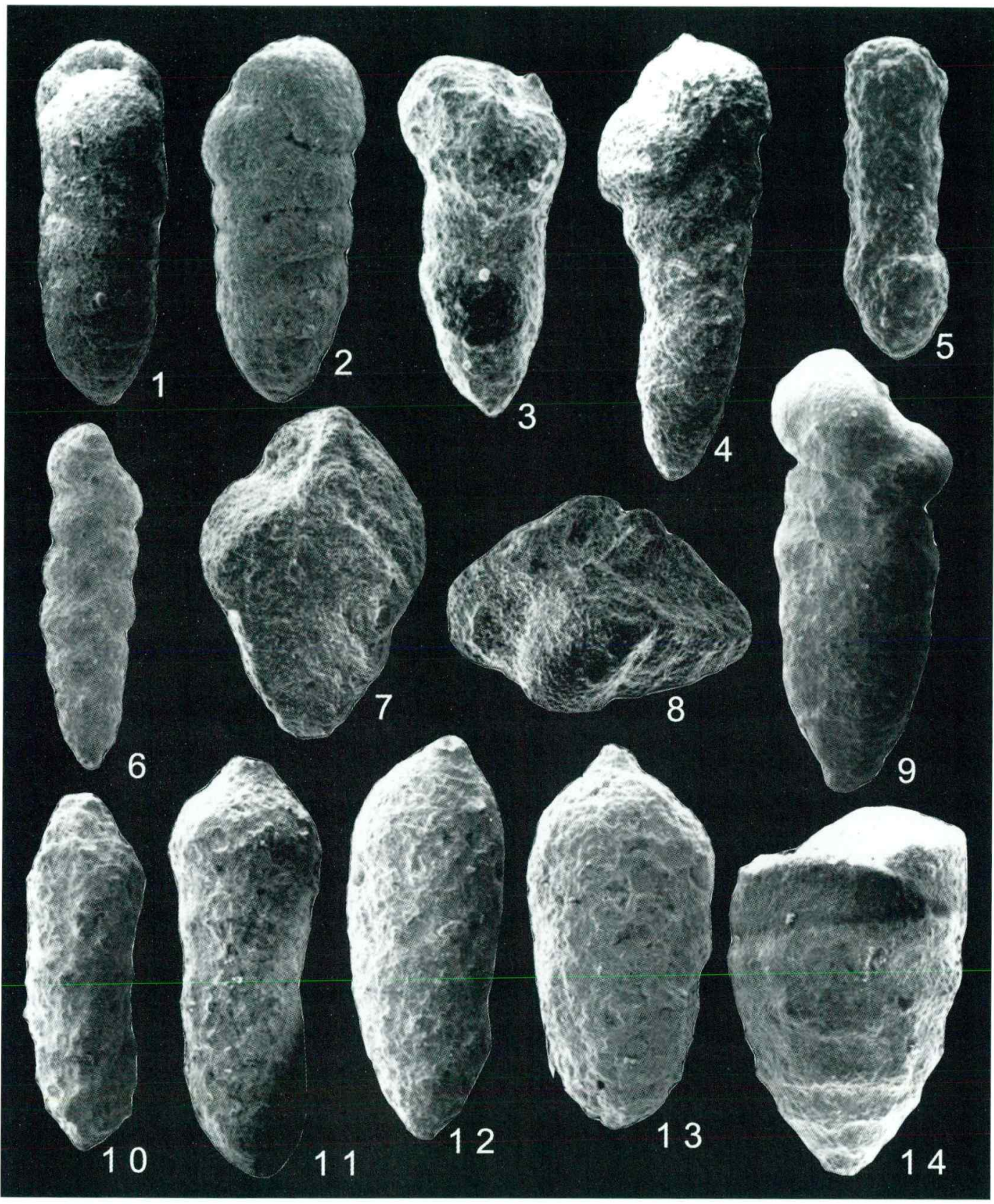

1-2 Dorothia fallax Hagn. 1 Zv/II-011, x40. 2 Zv/II-011, x40. 3-4 Karreriella halkyardi Cushman. 3 Vr010, x80. 4 Zv/II-006, x60. 5 Martinottiella sp., Vr-004, x80. 6 Textularia adalta Cushman, Vr-009, x40. 7-8 Karrerotextularia olianensis (Ruiz de Gaona \& Colom). 7 Vr-009, x120. 8 Vr-009, x120. 9 Pseudogaudryina textilaroides (Hantken), Vr-009, x28. 10-11 Cylindroclavulina colomi Hagn. 10 Vr-009, x40. 11 Vr-012, x40. 12-13 Cylindroclavulina rudislosta (Hantken). 12 Vr-009, x28. 13 Vr-009, x28. 14 Tritaxilina pupa (Gümbel), Vr-005A, x60. 
1993 Pseudogaudryina? textilaroides Hantken Sztràkos (In: Mathelin \& Sztràkos), p. 41, pl. 4 , fig. 5

Locality: Vr-009

Family: Valvulinidae Berthelin, 1880

\section{Cylindroclavulina colomi Hagn, 1956}

Pl. 2, Figs. 10-11

1956 Cylindroclavulina colomi - Hagn, p. 123, pl. 10, figs. 6-7; pl. 18, figs. 8. 9

1975 Cylindroclavulina colomi Hagn - Braga \& al. p. 103 , pl. 4 , fig. 6

1985 Cylindroclavulina colomi Hagn - Grünig, p. 258, pl. 4 , fig. 5

1993 Cylindroclavulina colomi Hagn - Sztràkos (In: Mathelin \& Sztràkos), p. 73, pl. 4, fig. 2

Locality: Vr-004, Vr-005A, Vr-009, Vr-

012, Vr-014, Zv-006, Zv-007

\section{Cylindroclavulina rudislosta (Hantken), 1889}

Pl. 2, Figs. 12-13

1889 Clavulina rudislosta - Hantken, p. 383

1875 Clavulina cylindrica Hantken (non d'Orbigny 1825) - Hantken, p. 18, pl. 1, fig. 8

1956 Cylindroclavulina rudislosta (Hantken) Hagn, p. 122, pl. 10, fig. 5

1991 Cylindroclavulina rudislosta (Hantken) Barbin \& Keller-Grünig, p. 240

1993 Cylindroclavulina rudislosta (Hantken) Sztràkos (In: Mathelin \& Sztràkos), p. 73, pl. 4 , fig. 4

Locality: Vr-004, Vr-005, Vr-005A, Vr009, Vr-012, Vr-014, Zv-004, Zv-005, Zv007

\section{Tritaxilina pupa (Gümbel), 1868}

Pl. 2, Fig. 14

1868 Gaudryina pupa - Gümbel, p. 602, pl. 1, fig. 3

1950 Tritaxilina pupa (Gümbel) - Ruiz de Gaona \& Colom, p. 421, fig. 14/23

1975 Tritaxilina pupa (Gümbel) - Braga et al., p. 93, pl. 1, figs. 24,27

1987a Tritaxilina pupa (Gümbel) - Sztràkos, p. 35, pl. 3, figs. 11-12

Locality: Vr-005A, Vr-011, Vr-014, Zv008, Zv-009, Zv-010, Zv-011

\section{Family: CornusPiridae Schultze, 1854}

\section{Cornuspira angigyra (Reuss), 1850}

Pl. 3, Fig. 1

1850 Operculina angigyra - Reuss, p. 370, pl. 46, fig. 19
1993 Cornuspira angigyra (Reuss) - Sztràkos (In: Mathelin \& Sztràkos), p. 74, pl. 4, fig. 13

Locality: Zv-006

Family: Spiroloculinidae Wiesner, 1920

\section{Spiroloculina dorsata Reuss, 1866}

1866 Spiroloculina dorsata - Reuss, p. 123

1855 Spiroloculina limbata - Bornemann, p. 348, pl. 19, fig. 1

1961 Spiroloculina canaliculata d'Orbigny Kaasschieter, p. 154, pl. 3, figs. 20-23

Locality: Vr-004, Vr-005A

\section{Spiroloculina sp. 1}

Pl. 3, Figs. 2-3

Locality: Vr-004, Vr-005, Vr-005A, Zv-

005, Zv-010, Zv-011

\section{Spiroloculina sp. 2}

Pl. 3, Fig. 4

Locality: Vr-005, Vr-011, Zv-006, Zv-007,

Zv-008, Zv-009, Zv-010, Zv-011

Family: Hauerinidae Schwager, 1876

Quinqueloculina depressa d'Orbigny, 1826 Pl. 3, Fig. 5

1826 Quinqueloculina depressa - d'Orbigny, p. 302, no. 38

1878 Triloculina depressa d'Orbigny - Terquem, pl. 8, figs. 1-11

1904 Quinqueloculina depressa d'Orbigny Fornasini, p. 68, pl. 4, fig. 8

1993 Quinqueloculina depressa d'Orbigny Sztràkos (In: Mathelin \& Sztràkos), p. 74, pl. 5, fig. 4

Locality: Vr-005A, Zv-006, Zv-009, Zv-

010, Zv-011

\section{Quinqueloculina impressa Reuss, 1851}

Pl. 3, Fig. 6

1851 Quinqueloculina impressa-Reuss, p. 87, pl. 7 , fig. 59

1993 Quinqueloculina impressa Reuss - Sztràkos (In: Mathelin \& Sztràkos), p. 74, pl. 5, fig. 6

Locality: Vr-004, Vr-005, Vr-005A, Vr-

009, Vr-010, Vr-011, Vr-011A, Vr-014, Zv004, Zv-005, Zv-006, Zv-007, Zv-008, Zv009, Zv-010, Zv-011

\section{Quinqueloculina sp. 1}

Pl. 3, Figs. 7-8

Locality: Vr-004, Vr-011, Vr-011A, Zv-

006, Zv-007, Zv-009, Zv-010, Zv-011 
PLATE 3

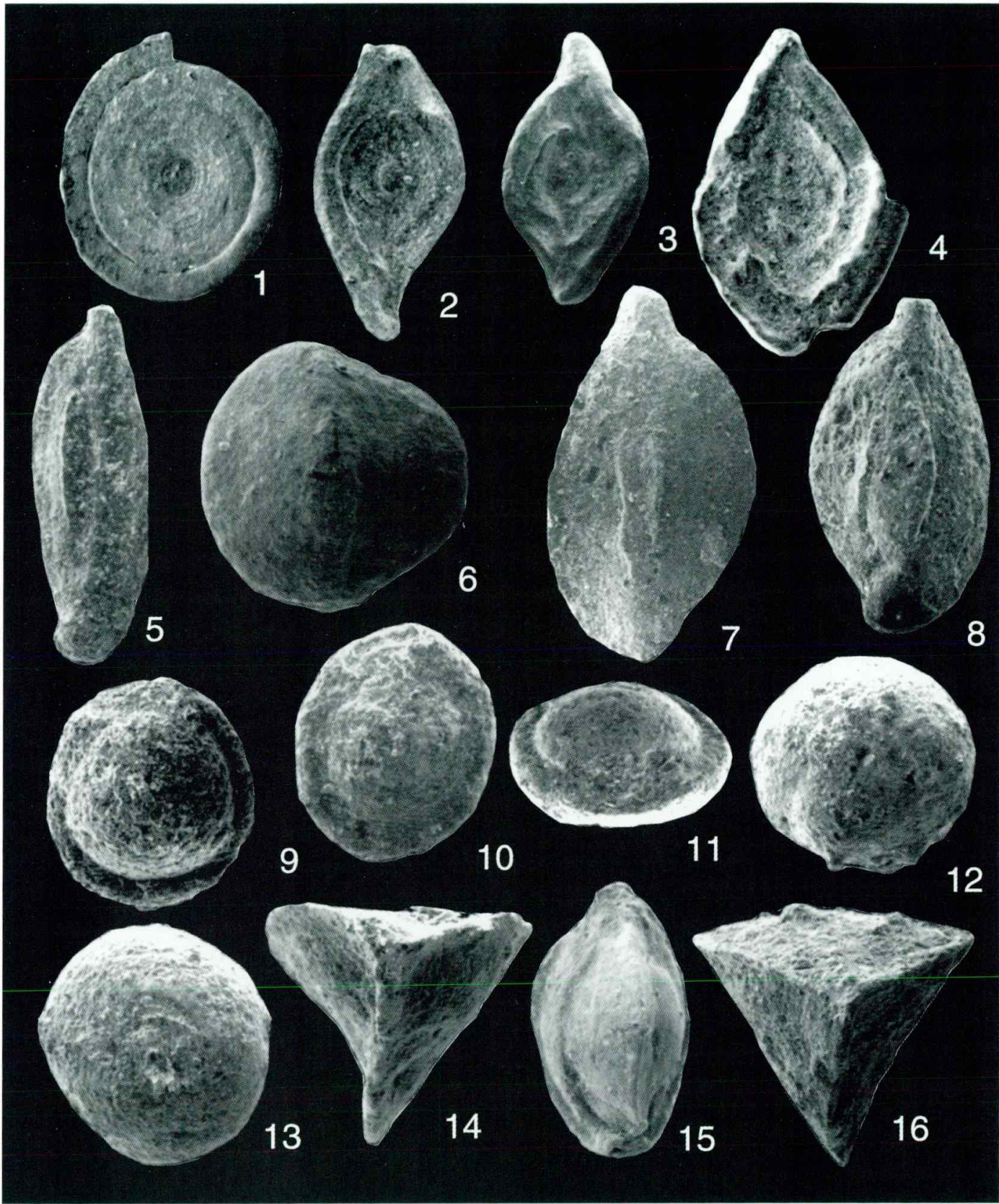

1 Cornuspira angigyra (Reuss), Zv/II-006, x40. 2-3 Spiroloculina sp. 1. 2 Zv/II-011, x40. 3 Zv/II-011, x40. 4 Spiroloculina sp. 2. Vr-010, x60 5 Quinqueloculina depressa d'Orbigny, Zv/II-010, x60. 6 Quinqueloculina impressa Reuss, Vr-009, x60. 7-8 Quinqueloculina sp. 1. 7 Zv/II-009, x60. 8 Zv/II-009, x60. 9 Pyrgo simplex (d'Orbigny), Zv/II-006, x80. 10-11 Pyrgo sp. 1. 10 Zv/II-005, x80. 11 Zv/II-006, x80. 12-13 Pyrgo sp. 2. 12 Zv/II-009, x80. 13 Pyrgo sp. 2, Zv/II-009, x80. 14-16 Nov. gen. sp. 1. 14 Zv/II-005, x120. 15 Zv/ II-010, x60. $16 \mathrm{Zv} / \mathrm{II}-007, \mathrm{x} 80$. 
Quinqueloculina sp. 2

Locality: Vr-011A, Zv-006, Zv-007, Zv009, Zv-010, Zv-011

\section{Quinqueloculina sp. 3}

Pl. 4, Fig. 5

Locality: Vr-003

\section{Pyrgo simplex (d'Orbigny), 1846}

Pl. 3, Fig. 9

1846 Biloculina simplex - d'Orbigny, p. 264, pl. 15 , figs. $25-27$

1975 Pyrgo simplex (d'Orbigny) - Braga et al., p. 104

1985 Pyrgo simplex (d'Orbigny) - Grünig, p. 260 fig. 9

1993 Pyrgo simplex (d'Orbigny) - Sztràkos (In: Mathelin \& Sztràkos), p. 74, pl. 5, fig. 9

Locality: Vr-012, Zv-005, Zv-006, Zv-008

\section{Pyrgo subsphaerica (d'Orbigny), 1839}

Pl. 4, Fig.6

1839 Biloculina subsphaerica - d'Orbigny, p. 162, pl. 8, figs. 25-27

1949 Pyrgo subsphaerica (d'Orbigny) - Cuvillier \& Szakall, p. 45 , pl. 20, fig. 2

1985 Pyrgo subsphaerica (d'Orbigny) - Grünig, p. 260

Locality: Vr-003

Pyrgo sp. 1

Pl. 3, Figs. 10-11

Locality: Zv-005, Zv-006

Pyrgo sp. 2

Pl. 3, Figs. 12-13

Locality: Zv-009, Zv-010, Zv-011

Nov. gen. sp. 1

Pl. 3, Figs. 14-16

Locality: Vr-004, Vr-005A, Zv-005, Zv-

006, Zv-007, Zv-008, ZV-010, ZV-011

\section{Sigmoilopsis bartoniensis (Ruiz de Gaona}

\& Colom), 1950

Pl. 4, Figs. 1-2

1951 Sigmoilina bartoniensis - Ruiz de Gaona \& Colom, p. 410, figs. 17/1-16

1985 Sigmoilopsis sp. - Grünig, p. 260, pl. 4, figs. 14-16

1993 Sigmoilopsis bartoniensis Ruiz de Gaona \& Colom - Sztràkos (In: Mathelin \& Sztràkos), p. 74 , pl. 5, fig. 12

Locality: Vr-011, Vr-011A, Zv-005, Zv-
006, Zv-007, Zv-008, Zv-009, Zv-010, Zv011

Family: Alveolinidae Ehrenberg, 1839

\section{Borelis vonderschmitti (Schweighauser),} 1951

Pl. 4, Figs. 3-4

1951 Neoalveolina vonderschmitti Schweighauser, p. 468, figs. 1-4

1979 Borelis aff. vonderschmitti (Schweighauser) - Drobne K. et al., p. 160, pl. 4, figs. 1-2

1990 Borelis vonderschmitti (Schweighauser) Pavlovec et al., p. 442, pl.1, figs. 1-3

Locality: Vr-005, Vr-005A

Family: Nodosariidae Ehrenberg, 1838

\section{Nodosaria latejugata Gümbel, 1868}

Pl. 4, Fig. 11

1868 Nodosaria latejugata - Gümbel, p. 41, pl. 1, fig. 32

1956 Nodosaria latejugata Gümbel - Hagn, p. 137, pl. 12 , fig. 11 ; pl. 13, fig. 1

1987a Nodosaria latejugata Gümbel - Sztràkos, p 33

1991 Nodosaria latejugata Gümbel - Barbin \& Keller-Grünig, p. 240

Locality: Vr-004, Vr-005 Vr-010, Vr-011,

Vr-012, Zv-008

\section{Pyramidulina bactridium (Reuss), 1866}

Pl. 4, Figs. 8-9

1866 Nodosaria bactridium - Reuss, p. 130, pl. 1, figs. 24-25

1993 Pyramidulina bactridium (Reuss) - Sztràkos (In: Mathelin \& Sztràkos). p. 75, pl. 7, figs. 15-16

Locality: Vr-005A

Family: Vaginulinidae Reuss, 1860

Lenticulina arcuatostriata (Hantken), 1868 Pl. 4, Fig. 13

1868 Cristellaria (Robulina) arcuato striata Hantken, p. 93, pl. 2, fig. 30

1950 Robulus arcuatostriatus (Hantken) - Ruiz de Gaona \& Colom, p. 402, figs. 6/1-9

1956 Robulus arcuato-striatus (Hantken) - Hagn, p. 127 , pl. 11, fig. 4

1975 Lenticulina arcuatostriata (Hantken) Braga et al., p. 104

1985 Lenticulina arcuato-striata (Hantken) Grünig, p. 261, pl. 4, fig. 19

Locality: Vr-005A, Vr-010, Vr-011, Zv-

005, Zv-010 
PLATE 4

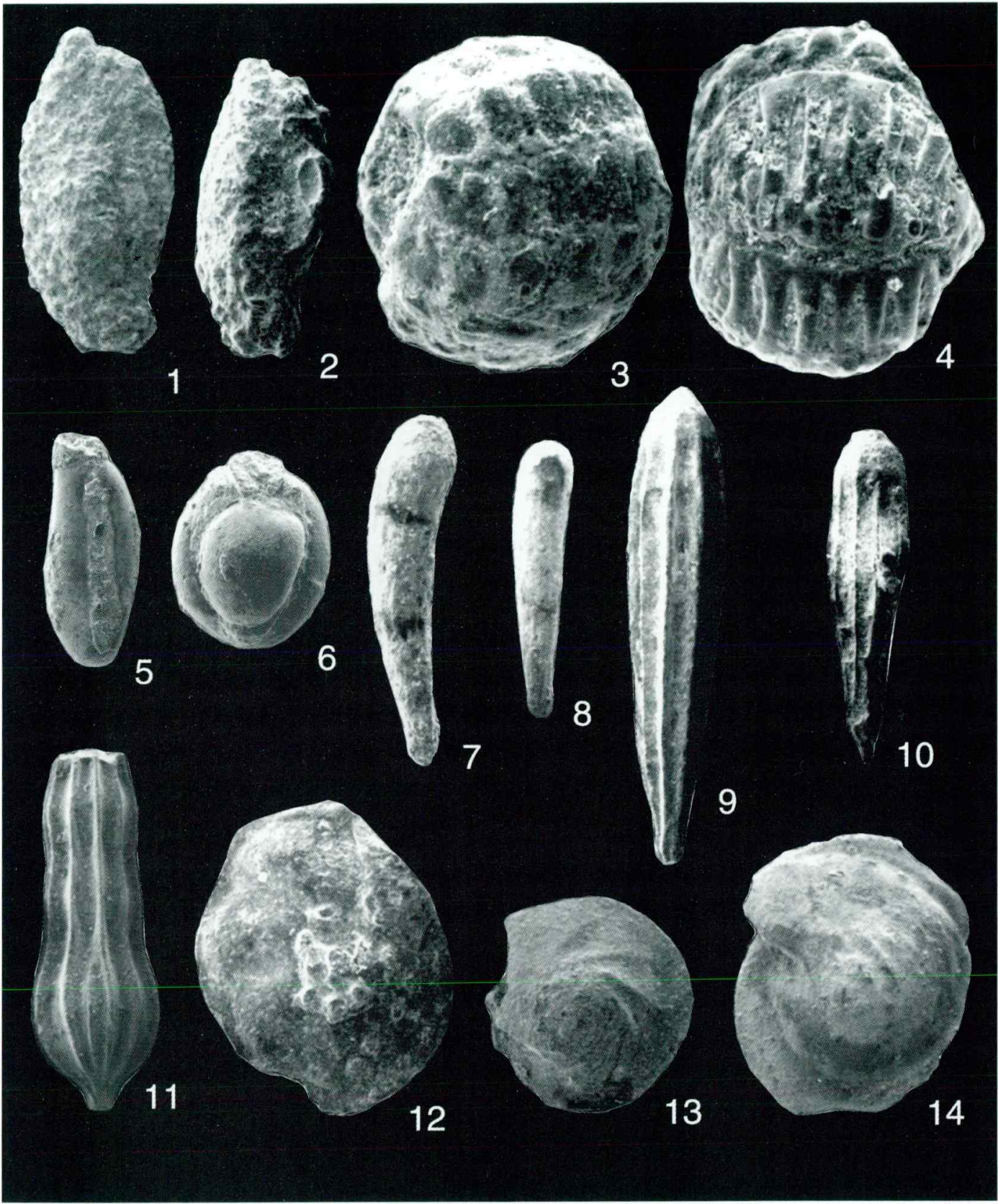

1-2 Sigmoilopsis bartoniensis (Ruiz de Gaona \& Colom). 1 Zv/II-010, x40. 2 Vr-011A, x40. 3-4 Borelis vonderschmitti (Schweighauser). $3 \mathrm{Vr}-005 \mathrm{~A}, \mathrm{x} 160.4 \mathrm{Vr}-005 \mathrm{~A}, \mathrm{x} 120.5$ Quinqueloculina sp. 3, Vr/II-003, x40 6 Pyrgo subsphaerica (d'Orbigny), Vr/II-003, x80. 7-8 Rectobolivina zsigmondyi (Hantken). 7 Zv/II009, x60. 8 Zv/II-009, x40. 9-10 Pyramidulina bactridium (Reuss). 9 Vr-005A, x28. 10 Vr-005A, x40. 11 Nodosaria latejugata Gümbel, Zv/II-006, x28. 12 Lenticulina budensis (Hantken), Vr-012, x40. 13 Lenticulina arcuatostriata (Hantken), Vr-011, x28. 14 Lenticulina limbosa (Reuss), Zv/II-008, x28. 
Lenticulina budensis (Hantken), 1875

Pl. 4, Fig. 12

1875 Robulina budensis - Hantken, p. 58, pl. 7, fig. 1

1950 Robulus budensis (Hantken) - Ruiz de Gaona \& Colom, p. 403, figs. 7/7-8

Locality: Vr-010, Vr-012

\section{Lenticulina limbosa (Reuss), 1863}

Pl. 4, Fig. 14

1863 Robulina limbosa - Reuss, p. 55, pl. 6, fig. $69 \mathrm{a}-\mathrm{b}$

1875 Robulina limbosa Reuss - Hantken, p. 57, pl. 6, fig. 11

1950 Rubulus limbosus (Reuss) - Ruiz de Gaona \& Colom, p. 405, fig. 5/1-14

1956 Robulus limbosus (Reuss) - Hagn, p. 127, pl. 11, fig. 2

1993 Lenticulina limbosa (Reuss) - Sztràkos (In Mathelin \& Sztràkos), p. 75, pl. 8, fig. 6

Locality: Zv-008

\section{Marginulinopsis behmi (Reuss), 1866}

Pl. 5, Fig. 1

1866 Cristellaria behmi - Reuss, p. 22, pl. 2, fig. 37

1868 Cristellaria behmi Reuss - Gümbel, s. 55, pl. 1, fig. 61

1875 Marginulina behmi (Reuss) - Hantken, p. 48 , pl. 5, figs. $1-2$

1950 Marginulina behmi (Reuss) - Ruiz de Gaona \& Colom, p. 384, fig. 11/18-26

1956 Marginulina behmi (Reuss) - Hagn, p. 131, pl. 11, fig. 11

1975 Marginulina behmi (Reuss) - Braga et al., p. 105, pl. 4, fig. 13

1985 Marginulina behmi (Reuss) - Grünig, p. 262, pl. 5, figs. 17-19

1993 Marginulina behmi (Reuss) - Sztràkos (In: Mathelin \& Sztràkos), p. 43, pl. 26, figs. 1416

Locality: Vr-004, Vr-005, Vr-009, Vr-010, Vr-011, Vr-012, Vr-014, Zv-006, Zv-008, Zv-009, Zv-010, Zv-011

\section{Marginulinopsis porvaensis (Hantken), 1875}

Pl. 5, Figs. 2-3

1875 Cristellaria porvaensis - Hantken, p. 50, pl. 14, fig. 1

1950 Marginulina porvaensis (Hatken) - Ruiz de Gaona \& Colom, p. 387, fig. 11/1-14

1987aMarginulinopsis porvaensis (Hantken) Sztràkos, p. 32, pl. 5, fig. 20

1993 Astacolus porvaensis (Hantken) - Sztràkos (In: Mathelin \& Sztràkos), p. 76, pl. 26, fig. 13
Locality: Vr-010, Vr-011, Zv-005, Zv-007, $\mathrm{Zv-008,} \mathrm{Zv-009,} \mathrm{Zv-010}$

Percultazonaria fragaria (Gümbel), 1868

Pl. 5, figs. 4-5

1868 Marginulina fragaria - Gümbel, p. 57, pl. 1, figs. $58 \mathrm{a}-\mathrm{c}$

1950 Marginulina fragaria - Ruiz de Gaona \& Colom, p. 386, figs. $11 / 15-17$

1956 Marginulinopsis fragaria (Gümbel) - Hagn, p. 134 , pl. 12 , figs. $3,8,10$

1975 Marginulinopsis fragaria (Gümbel) - Braga et al., p. 105

1985 Marginulinopsis fragaria (Gümbel) - Grünig, p. 161 , pl. 5, figs. 24-28

1991 Marginulinopsis fragaria (Gümbel) - Barbin \& Keller-Grünig, p. 240, pl. 2, figs. 6-12

1993 Percultazonaria fragaria (Gümbel) Sztràkos (In: Mathelin \& Sztràkos), p. 76 pl. 9, figs. 1-2

Locality: Vr-014, Zv-004, Zv-006, Zv-007, Zv-009

\section{Saracenaria hantkeni Cushman, 1933}

Pl. 5, Fig. 6

1933 Saracenaria arcuata d'Orbigny var. hantkeni - Cushman, p. 4, pl. 1, figs. 11-12 (Fide Ellis \& Messina 1940)

1956 Saracenaria hantkeni Cushman - Hagn, p. 138 , pl. 13, fig. 4

1993 Saracenaria hantkeni Cushman - Sztràkos (In: Mathelin \& Sztràkos), p. 76, pl. 9, fig. 6

Locality: Vr-004, Vr-009, Vr-010, Zv-005,

Zv-006, Zv-007, Zv-010, Zv-011

\section{Palmula budensis (Hantken), 1875}

Pl. 5, Fig. 7

1875 Flabellina budensis - Hantken, p. 44, pl. 4, fig. 17

1984 Frondicularia budensis (Hantken) - Grünig \& Herb, p. 263

1985 Frondicularia budensis (Hantken) - Grünig, p. 261, pl. 4, fig. 21

1993 Palmula budensis (Hantken) - Sztràkos (In: Mathelin \& Sztràkos), p. 76, pl. 9, fig. 10

Locality: Vr-011, Zv-005, Zv-006, Zv-007, Zv-008

\section{Palmula tenuissima (Hantken), 1875}

Pl. 5, Figs. 8-9

1875 Frondicularia tenuissima - Hantken, p. 43, pl. 13 , fig. 11

1950 Frondicularia tenuissima Hantken - Ruiz de Gaona \& Colom, p. 377 
PLATE 5

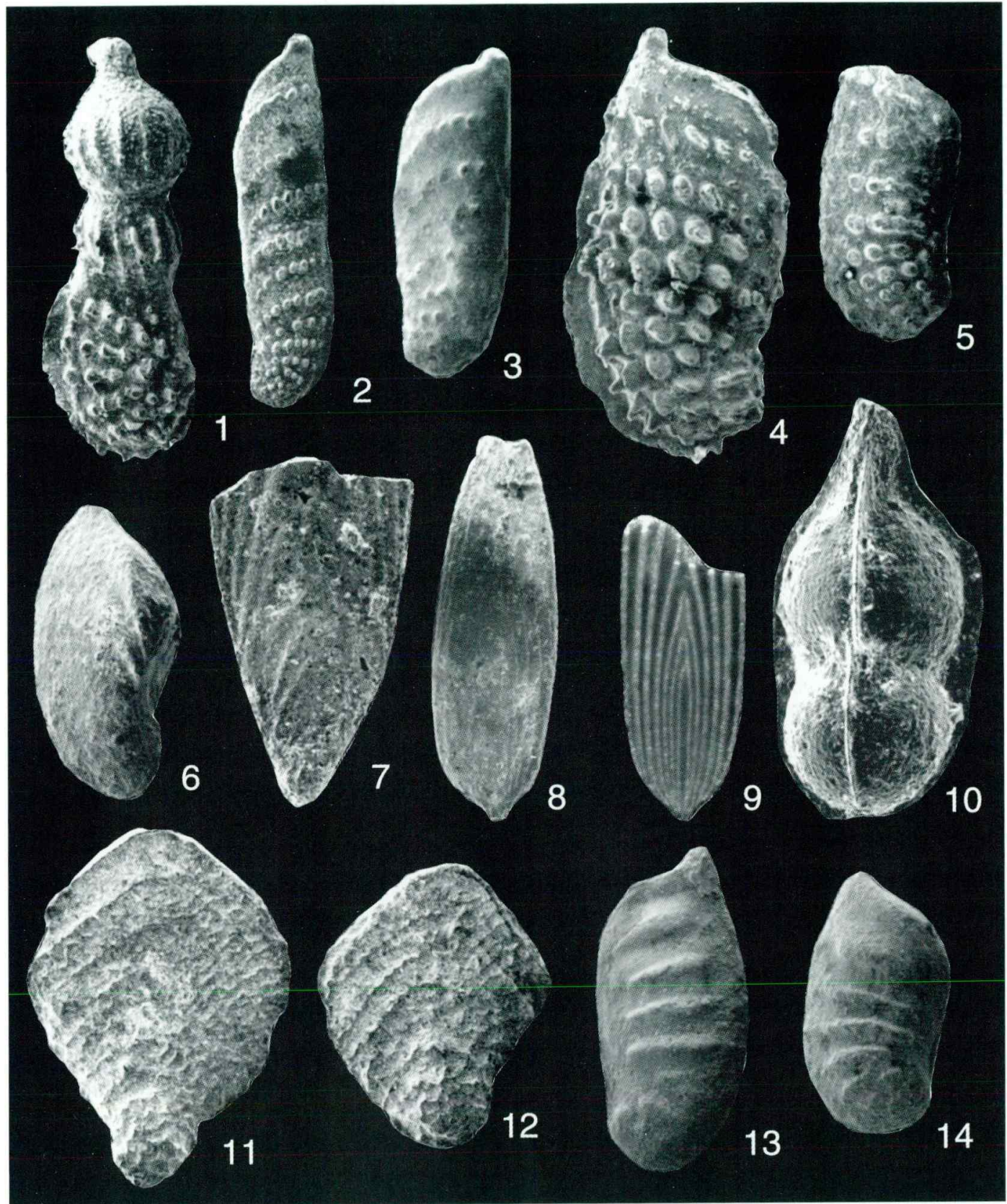

1 Marginulinopsis behmi (Reuss), Zv/II-006, x60. 2-3 Marginulinopsis porvaensis (Hantken). 2 Zv/II-006, x28. 3 Zv/II-010, x40. 4-5 Percultazonaria fragaria (Gümbel). 4 Zv/II-006, x40. 5 Vr-012, x28. 6 Saracenaria hantkeni (Cushman), Vr-010, x40. 7 Palmula budensis (Hantken), Zv/II-006, x80. 8-9 Palmula tenuissima (Hantken). 8 Zv/II-008, x80. 9 Zv/II-005, x60. 10 Marginulina globosa (Halkyard), Zv/II-006, x120. 1112 Reticulopalmula arborescens (Halkyard). 11 Vr-005, x40. 12 Vr-005, x40. 13-14 Vaginulinopsis cumulicostata (Gümbel). 13 Vr-004, x28. 14 Vr-004, x28. 
1993 Frondovaginulina tenuissima (Hantken) Sztràkos (In: Mathelin \& Sztràkos), p. 76, pl. 9 , fig. 8

Locality: Vr-005A, Zv-008

\section{Reticulopalmula arborescens (Halkyard), 1919}

Pl. 5, Figs. 11-12

1919 Frondicularia arborescens - Halkyard, p. 88, pl. 5, figs. $8-9$

1981 Coleites arborescens (Halkyard) - Hagn et al., p. 91

1985 Coleites arborescens (Halkyard) - Grünig, p. 275, pl. 6, figs. 14-15

1993 Reticulopalmula arborescens (Halkyard) Sztràkos (In: Mathelin \& Sztràkos), p. 43, pl. 26, figs. 8-9

Locality: Vr-005, Vr-009, Vr-010, Vr-011,

Vr-012, Zv-005, Zv-006, Zv-008 Zv-010, Zv-011

\section{Marginulina globosa Halkyard, 1919}

Pl. 5, Fig. 10

1919 Marginulina pyramidale Karrer var. globosa - Halkyard, p. 85, pl. 5, fig. 2

1993 Marginulina globosa Halkyard - Sztràkos (In: Mathelin \& Sztràkos), p. 44, pl. 23, fig. 15 ; pl. 48, figs. 20-22

Locality: Vr-011, Zv-006

\section{Vagunulinopsis cumulicostata (Gümbel), 1868}

Pl. 5, Figs. 13-14

1868 Cristellaria cumulicostata - Gümbel, p. 60, pl. 1, fig. 67

1956 Vaginulinopsis cumulicostata (Gümbel) Hagn, p. 135, pl. 13, fig. 3

1975 Vaginulinopsis cumulicostata (Gümbel) Braga et al. p. 105, pl. 4, fig. 8

1985 Vaginulinopsis cumulicostata (Gümbel) Grünig, p. 263, pl. 6, fig. 6

1993 Vaginulinopsis cumulicostatus (Gümbel) Sztràkos (In: Mathelin \& Sztràkos), p. 76, pl. 10 , fig. 12

Locality: Vr-004, Vr-005, Vr-010, Vr-012, Zv-005

\section{Spirolingulina acutimargo (Halkyard), \\ 1919}

Pl. 6, Fig. 1

1919 Lingulinopsis acutimargo - Halkyard, p. 81, pl. 5 , fig. 1

1949 Lingulina acutimargo (Halkyard) - Cuvillier \& Szakall, p. 81, pl. 29, fig. 11

1950 Lingulina acutimargo (Halkyard) - Ruiz de Gaona \& Colom, p. 384, fig. 11/31-3
1993 Spirolingulina acutimargo (Halkyard) Sztràkos (In: Mathelin \& Sztràkos), p. 44, pl. 27, fig. 2

Locality: Vr-004, Zv-009

Family: Ceratobuliminidae Cushman, 1927

Ceratocancris crassa (Halkyard), 1919

Pl. 6, Figs. 2-4

1919 Pulvinulina haueri d'Orbigny var. crassa Halkyard, p. 125, pl. 7, fig. 6

1957 Ceretobulimina haueri d'Orbigny var. crassa (Halkyard) - Sacal \& Debourle, p. 46, pl. 20, fig. 1

1993 Ceratocancris crassa (Halkyard) - Sztràkos (In: Mathelin \& Sztràkos), p. 45, pl. 11, fig. 17

Locality: Vr-014

\section{Lamarckina cristellaroides (Terquem), 1882}

Pl. 6, Fig. 7

1882 Rotalina cristellaroides - Terquem, p. 57, pl. 3 , fig. $15 \mathrm{a}-\mathrm{c}$

1961 Lamarckina cristellaroides (Terquem) Kaasschieter, p. 231, pl. 15, figs. 3-4

1970 Lamarckina cristellaroides (Terquem) - Le Calvez, p. 203, pl. 37, fig. 4

1993 Lamarckina cristellaroides (Terquem) Sztràkos (In: Mathelin \& Sztràkos), p. 77, pl. 11, fig. 19 ; pl. 29, fig. 6

Locality: Vr-005A

Family: Epistominidae Wedekind, 1937

\section{Hoeglundina eocenica (Cushman \&}

Hanna), 1927

Pl. 6, Figs. 5-6

1927 Epistomina eocenica - Cushman \& Hanna, p. 53 , pl. 5, figs. $4-5$

1975 Hoeglundina eocenica (Cushman \& Hanna) - Braga et al., p. 110

1981 Hoeglundina eocenica (Cushman \& Hanna) - Lindenberg et al., p. 148

1985 Hoeglundina eocenica (Cushman \& Hanna) - Grünig, p. 277, pl. 11, figs. 22-24

1987a Hoeglundina eocenica (Cushman \& Hanna) - Sztràkos, p. 31, pl. 15, fig. 9

Locality: Vr-005, Vr-010, Vr-011A, Vr012, Zv-010

Family: Bolivinidae Glaesner, 1937

\section{Bolivina nobilis Hantken, 1875}

Pl. 6, Figs. 9-10

1875 Bolivina nobilis - Hantken, p. 65, pl. 15, fig. $4 \mathrm{a}-\mathrm{b}$ 


\section{PLATE 6}

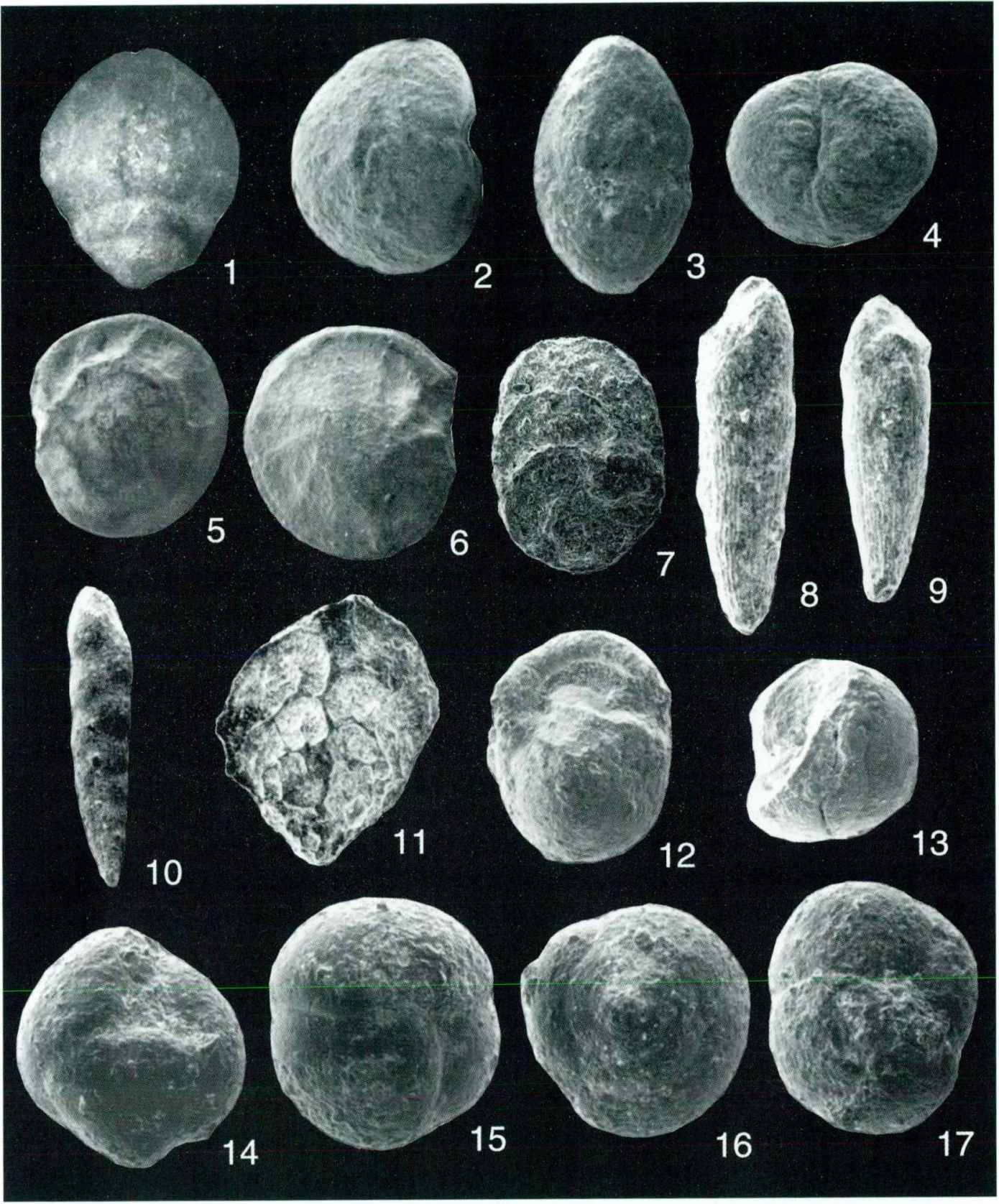

1 Spirolingulina acutimargo (Halkyard), Vr-004, x40. 2-4 Ceratocancris crassa (Halkyard). 2 spiral view Vr-014, x40. 3 edge view, Vr-014, x40. 4 umbilical view, Vr-014, x40. 5-6 Hoeglundina eocenica (Cushman \& Hanna). 5 spiral view, Vr-010, x28. 6 umbilical view, Vr-010, x28. 7 Lamarckina cristellaroides (Terquem), Vr-005A. 8-9 Bolivina nobilis Hantken. 8 Vr-010, x80. 9 Vr-010, x80. 10 Bolivina semistriata Hantken, Vr005A, x40. 11 Aragonia janoscheki Gohrbandt, Vr-010, x120. 12-13 Globocassidulina globosa (Hantken). 12 Vr.011, x80. 13 Vr-009, x80. 14-17 Sporobulimina eocaena Bykova. 14 side view, Vr-008, x120. 15 apertural view, Vr-005A, x120. 16 apical view, Zv/II-008, x120. 17 apertural view, Zv/II-008, x120. 
1950 Bolivina nobilis Hantken - Ruiz de Gaona \& Colom, p. 364

1956 Bolivina nobilis Hantken - Hagn, p. 147

1975 Bolivina nobilis Hantken - Braga et al., p. 106, pl. 5, figs. 1-2

1985 Bolivina nobilis Hantken - Grünig, p. 265, pl. 5, figs. 12-14

1991 Bolivina nobilis Hantken - Barbin \& KellerGrünig, p. 240

1993 Bolivina nobilis Hantken - Sztràkos (In: Mathelin \& Sztràkos), p. 78, pl. 32, fig. 9 Locality: Vr-004, Vr-005, Vr-009, Zv-007, Zv-008, Zv-009

\section{Bolivina semistriata Hantken, 1875}

Pl. 6, Fig. 10

1875 Bolivina semistriata - Hantken, p. 65, pl. 7, fig. 13

1956 Bolivina semistriata Hantken - Hagn, p. 147

1993 Bolivina semistriata Hantken - Sztràkos (In: Mathelin \& Sztràkos), p. 78, pl. 32, fig. 10 Locality: Vr-011, Vr-012, Zv-005, Zv-006, Zv-010

Bolivina pl. sp.

Locality: Vr-005, Vr-009, Vr-011, Vr-012, Zv-005, Zv-007, Zv-008, Zv-009, Zv-010, Zv-011

Family: Loxostomatidae Loeblich \& Tappan, 1962

\section{Aragonia janoscheki Gohrbandt, 1962}

Pl. 6, Fig. 11

1962 Aragonia janoscheki-Gohrbandt, p. 81, pl. 3 , figs. $5 \mathrm{a}-\mathrm{b}$

1975 Aragonia janoscheki Gohrbandt - Braga et al., p. 106 , pl. 5, fig. 7

Locality: Vr-010, Vr-012

\section{Family: Cassidulinidae d'Orbigny, 1839}

\section{Globocassidulina globosa (Hantken), 1875}

Pl. 6, Figs. 12-13

1875 Cassidulina globosa-Hantken, p. 64, pl. 16, figs. $2 \mathrm{a}-\mathrm{b}$

1956 Cassidulina globosa Hantken - Hagn, p. 167, pl. 14, figs. 9-10

1975 Globocassidulina globosa (Hantken) - Braga et al., p. 108, pl. 6, fig. 4

1985 Globocassidulina globosa (Hantken) Grünig, p. 273, pl. 10, fig. 3

1987aGlobocassidulina globosa Hantken Sztràkos, p. 31, pl. 11, fig. 22

1993 Globocassidulina globosa (Hantken) Sztràkos (In: Mathelin \& Sztràkos), p. 79
Locality: Vr-004, Vr-005, Vr-005A, Vr009, Vr-011, Vr-011A

Family: Turrilinidae Cushman, 1927

Sporobulimina eocaena Bykova, 1959

Pl. 6, Figs. 14-17

1959 Sporobulimina eocaena - Bykova, p. 76, pl. 12, fig. 6a-b (Fide Ellis \& Messina 1940)

1993 Sporobulimina eocaena Bykova - Sztràkos (In: Mathelin \& Sztràkos), p. 46, pl. 33, figs. 11-12

Locality: Vr-005A, Zv/II-008

Family: Siphogenerinoididae Saidova, 1981

Rectobolivina zsigmondyi (Hantken), 1868 Pl. 4, Figs. 7-8

1868 Nodosaria (Dentalina) zsigmondyi Hantken, p. 87, pl. 1, fig. 12

1982 Rectobolivina zsigmondyi (Hantken) Sztràkos, pl. 15, fig. 14

1987 Rectobolivina zsigmondyi (Hantken) Reiser, p. 91, pl. 9, figs. 13, 19

Locality: Zv-005, Zv-008, Zv-009, Zv-010

Sagrinopsis aspera (Terquem), 1882

Pl. 7, Figs. 1-3

1882 Textilaria aspera - Terquem, p. 147, pl. 15, fig. 14 (Fide Ellis \& Messina 1940)

1919 Siphogenerina hexagona Halkyard Halkyard, p. 41, pl. 6, fig. 5a-b

1975 Bifarina aff. selseyensis (Heron-Allen \& Earland) - Braga et al., p. 108, pl. 5, fig. 9

1985 Rectobolivina sp. - Grünig, p. 266, pl. 8, fig. 22

1993 Sagrinopsis aspera (Terquem) - Sztràkos (In: Mathelin \& Sztràkos), p. 47, pl. 33, fig. 15

Locality: Vr-005, Vr-005A

\section{Tubulogenerina tubulifera (Parker \&} Jones), 1863

Pl. 7, Figs. 4-5

1863 Textularia tubulifera - Parker \& Jones, p. 94, fig. 2 (Fide Ellis \& Messina 1940)

1837 Tubulogenerina tubulifera (Parker \& Jones) - Cushman, p. 215, pl. 24, figs. 14-16

1970 Tubulogenerina tubulifera (Parker \& Jones) - Le Calvez, p. 122, pl. 26, fig. 7

1987a Tubulogenerina tubulifera (Parker \& Jones) - Sztràkos, p. 35, pl. 7, fig. 20; pl. 18, fig. 2

1991 Tubulogenerina tubulifera (Parker \& Jones) - Gibson et al., p. 307, pl. 1, figs. 1-11; pl. 3 , figs. $6-8$

Locality: Vr-005, Vr-005A 


\section{PLATE 7}

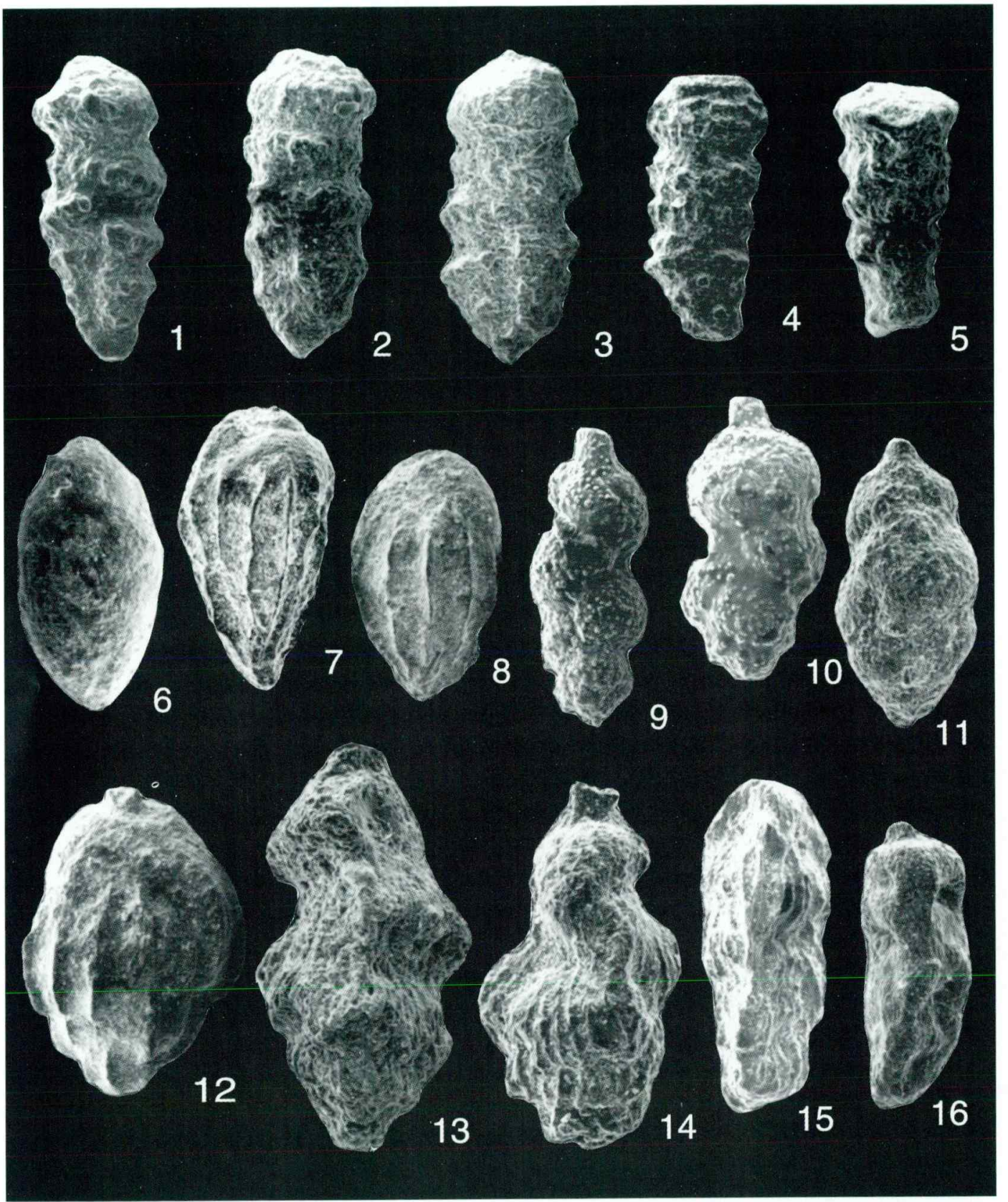

1-3 Sagrinopsis aspera (Terquem). 1 Vr-005, x80. 2 Vr-005, x120. 3 Vr-005A, x120. 4-5 Tubulogenerina cf tubulifera (Parker \& Jones). 4 Vr-005A, x120. 5 Vr-005, x120. 6 Bulimina ovata d'Orbigny, Vr-010, x60. 7 Bulimina subtruncana Hagn, Vr-010, x160. 8 Bulimina truncana Gümbel, Vr-005, x80. 9-10 Uvigerina gracilis Reuss. $9 \mathrm{Vr}-005, \mathrm{x} 160.10 \mathrm{Vr}-005, \mathrm{x} 160.11$ Uvigerina chirana Cushman \& Stone, Vr005, x120. 12 Uvigerina eocaena Gümbel, Vr-010, x80. 13 Angulogerina muralis (Terquem), Zv/II-008, x160. 14 Angulogerina pulchella Cushman \& Edwards, Vr-005, x160. 15 Angulogerina globosa (Stoltz), Vr-014, x120. 16 Koleshnikovella elongata (Halkyard), Zv/II-010, x120. 
Family: Buliminidae Jones, 1875

Bulimina ovata d'Orbigny, 1846

Pl. 7, Fig. 6

1846 Bulimina ovata - d'Orbigny, p. 185, pl. 11, figs. 13-14

1961 Bulimina ovata d'Orbigny - Kaasschieter, p. 191 , pl. 9, fig. 6

1985 Bulimina ovata d'Orbigny - Grünig, p. 266, pl. 6, fig. 22

Locality: Vr-010

\section{Bulimina subtruncana Hagn, 1956}

Pl. 7, Fig. 7

1954 Bulimina subtruncana - Hagn, p. 17, pl. 3, fig. 19 ; pl. 4 , fig. 9

1985 Bulimina subtruncana Hagn - Grünig, p. 266, pl. 6, fig. 23

Locality: Vr-010

\section{Bulimina truncana Gümbel, 1868}

Pl. 7, Fig. 8

1868 Bulimina truncana - Gümbel, p. 66, pl. 2, fig. $77 \mathrm{a}-\mathrm{b}$

1975 Bulimina truncana Gümbel - Hantken, p. 61, pl. 7, fig. 5

1975 Bulimina truncana Gümbel - Braga et al., p. 106 , pl. 4 , figs. $15-16$

1985 Bulimina truncana Gümbel - Grünig, p. 267, pl. 7 , figs. $26-27$

Locality: Vr-005, Vr-005A, Vr-010, Vr-

011A, Zv-009

\section{Family: Uvigerinidae Haeckel, 1894}

\section{Uvigerina chirana Cushman \& Stone,}

\section{7}

Pl. 7, Fig. 11

1947 Uvigerina chirana - Cushman \& Stone, p. 17, pl. 2, fig. 25 (Fide Ellis \& Messina 1940)

1977 Uvigerina chirana Cushman \& Stone - Braga et al., p. 95, pl. 5, fig. 10

1985 Uvigerina chirana Cushman \& Stone Grünig, p. 267, pl. 7, figs. 3-5

Locality: Vr-005, Zv-004, Zv-005

\section{Uvigerina eocaena Gümbel, 1868}

Pl. 7, Fig. 12

1868 Uvigerina eocaena - Gümbel, p. 645, pl. 2, fig. 78

1975 Uvigerina eocaena Gümbel - Braga et al., p. 107, pl. 5, fig. 17

1985 Uvigerina eocaena Gümbel - Grünig, p. 267, pl. 7, figs. $8-10$
1993 Uvigerina eocaena Gümbel - Sztràkos (In: Mathelin \& Sztràkos), p. 79, pl. 34, figs. 910

Locality: Vr-004, Vr-010, Vr-011 Zv-005, Zv-006, Zv-008, Zv-010, Zv-011

\section{Uvigerina gracilis Reuss, 1851}

Pl. 7, Figs. 9-10

1851 Uvigerina gracilis - Reuss, p. 77, pl. 5, fig. 39

1993 Uvigerina gracilis Reuss - Sztràkos (In: Mathelin \& Sztràkos), p. 79, pl. 34, fig. 13

Locality: Vr-005

Angulogerina globosa (Stoltz), 1925

Pl. 7, Fig. 15

1925 Uvigerina tenuistriata var. globosa - Stoltz, p. 130 (Fide Ellis \& Messina 1940)

1993 Angulogerina globosa (Stoltz) - Sztràkos (In: Mathelin \& Sztràkos), p. 48, pl. 34, fig. 20

Locality: Vr-014

Angulogerina muralis (Terquem), 1882

Pl. 7, Fig. 13

1882 Uvigerina muralis - Terquem, p. 119, pl. 12, figs. 26-29 (Fide Ellis \& Messina 1940)

1919 Tritaxia dehiscens - Halkyard, p. 44, pl. 3, fig. 8

1985 Trifarina muralis (Terquem) - Grünig, p. 268, pl. 7, fig. 26

1993 Angulogerina muralis (Terquem) - Sztràkos (In: Mathelin \& Sztràkos), p. 79, pl. 43, fig. 18

Locality: Zv/II-008

\section{Angulogerina pulchella Cushman \&}

Edwards, 1937

Pl. 7, Fig. 14

1937 Angulogerina pulchella - Cushman \& Edwards, p. 61, pl. 8, fig. 19 (Fide Ellis \& Messina 1940)

1993 Angulogerina pulchella Cushman \& Edwards - Sztràkos (In: Mathelin \& Sztràkos), p. 48, pl. 34, fig. 17

Locality: Vr-005

\section{Trifarina sp. 1}

Locality: Vr-012

Koleshnikovella elongata (Halkyard), 1919 Pl. 7, Fig. 16

1919 Tritaxia elongata - Halkyard, p. 45, pl. 3, fig. 9

1987a Uvigerinella elongata (Halkyard) - Sztràkos, p. 36, pl. 18 , fig. 5

1993 Koleshnikovella elongata (Halkyard) Sztràkos (In: Mathelin \& Sztràkos). p. 48, pl. 34 , fig. 16 
PLATE 8

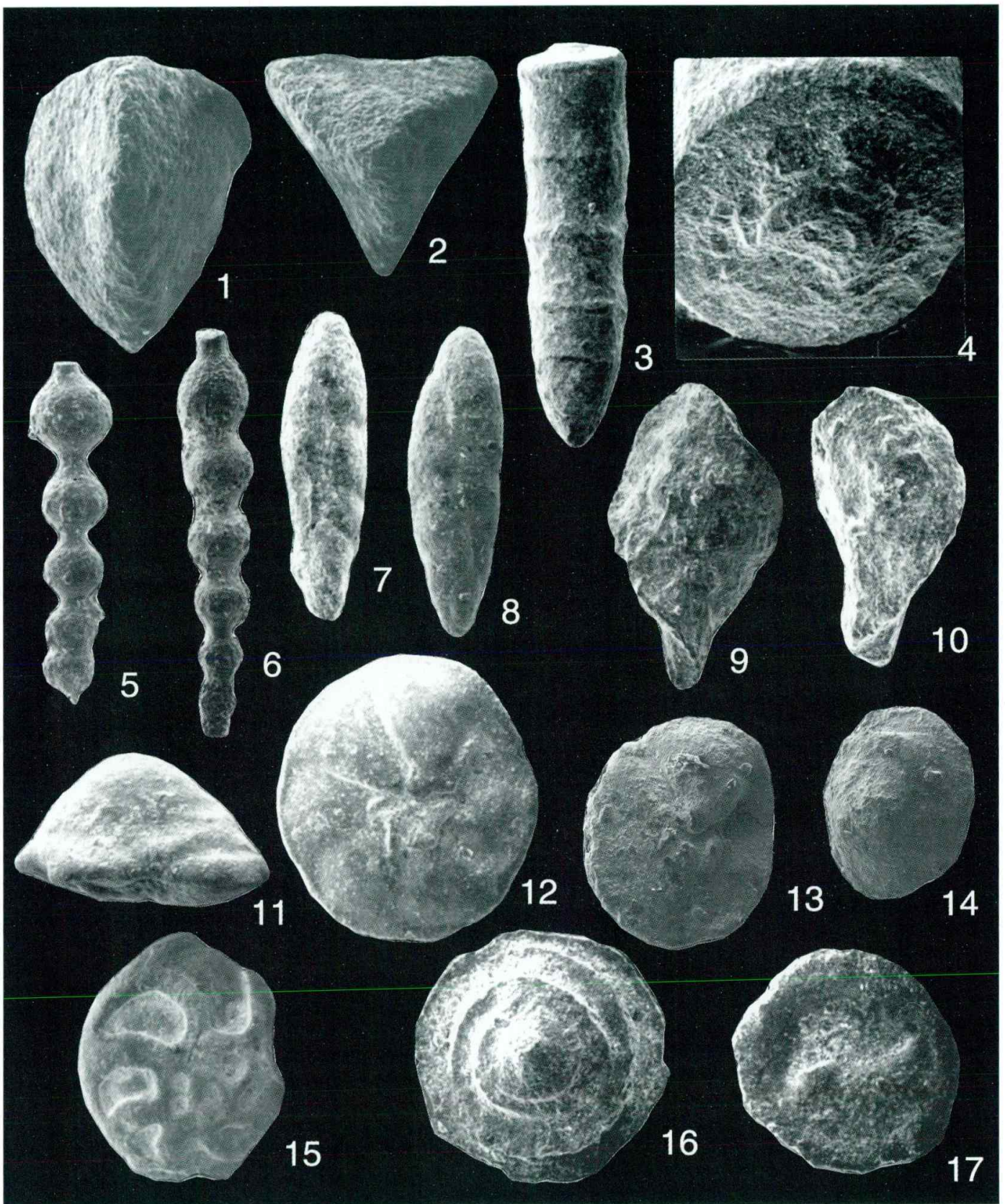

1-2 Reussella oberburgensis (Reuss). 1 Vr-005A, x120. 2 Vr-005A, x120. 3-4 Siphonodosaria crassisepta (Halkyard). 4 Vr-010, x40. 4 Vr-010, x40. 5-6 Nodogenerina atlantisae Cushman. 5 Zv/II-006, x80. 6 Zv/ II-008, x80. 7-8 Fursenkoina halkyardi (Cushman). 7 Vr-012, x60. 8 Zv/II-008, x60. 9-10 Sigmavirgulina tortuosa (Brady). 9 Vr-005, x80. 10 Vr-010, x120. 11-12 Neoeponides schreibersii (d'Orbigny). 11 Zv/II005, x40. 12, Zv/II-005, x40. 13-14 Eponides ouachitaensis Howe \& Wallace. 13 Vr-011A, x60. 14 Vr011A, x60. 15 Schlosserina asterites (Gümbel), Vr-014, x28. 16-17 Neoconorbina sp. 16 spiral view, Vr005A, x80. 17 umbilical view Vr-005A, x80. 
Locality: Zv-010

Family: Reussellidae Cushman, 1933

\section{Reussella oberburgensis (Reuss), 1864}

Pl. 8, Figs. 1-2

1864 Verneuilina oberburgensis Frey. in litt. Reuss, p. 6, pl. 1, fig. 2

1962 Reussella oberburgensis (Freyer) Gohrbandt, p. 82, pl. 3, fig. 6a-c

1985 Reussella oberburgensis (Freyer) - Grünig, p. 267

1993 Reussella oberburgensis (Freyer) - Sztràkos (In: Mathelin \& Sztràkos), p. 79, pl. 34, fig. 26

Locality: Vr-004, Vr-005, Vr-005A, Vr-011

Family: Fursenkoinidae Loeblich \&

TaPpan, 1961

\section{Fursenkoina halkyardi (Cushman), 1936}

Pl. 8, Figs. 7-8

1936 Virgulina halkyardi - Cushman, p. 47, pl. 7, fig. 5

1937b Virgulina halkyardi Cushman - Cushman, p. 11, pl. 1, figs. 26-27

1985 Fursenkoina halkyardi (Cushman) - Grünig, p. 273, pl. 9, figs. 25-27

1993 Fursenkoina halkyardi (Cushman) Sztràkos (In: Mathelin \& Sztràkos), p. 49, pl. 35 , fig. 6

Locality: Vr-005, Vr-012, Zv-007, Zv-009,

Zv-010, Zv-011

\section{Sigmavirgulina tortuosa (Brady), 1881}

Pl. 8, Figs. 9-10

1881 Bolivina tortuosa - Brady, p. 57 (Fide Ellis \& Messina 1940)

1960 Sigmavirgulina tortuosa (Brady) - Barker, pl. 52, figs. 31-32

Locality: Vr-005, Vr-005A, Vr-010, Vr-

011, Vr-011A, Vr-012, Zv-005

Family: Stilostomellidae Finlay, 1947

\section{Siphonodosaria crassisepta (Halkyard), 1919}

Pl. 8, Figs. 3-4

1919 Nodosaria pauperata d'Orbigny var. crassisepta - Halkyard, p. 72, pl. 4, figs. 1213

1941 Dentalina annulata (Reuss) - de Witt Puyt, p. 51 , pl. 1 , figs. $23-25$

1957 Nodogenerina crassisepta (Halkyard) Sacal \& Debourle, p. 16 , pl. 4, fig. 8
1993 Siphonodosaria crassisepta (Halkyard) Sztràkos (In: Mathelin \& Sztràkos), p. 51, pl. 14, figs. 3-11

Locality: Vr-004, Vr-005A, Vr-009, Vr-

010, Vr-014, Zv-005, Zv-006, Zv-007, Zv-

008, Zv-009, Zv-010

Family: Eponididae Hofker, 1951

\section{Eponides ouachitaensis Howe \& Wallace, 1932}

Pl. 8, Figs. 13-14

1932 Eponides ouachitaensis - Howe \& Wallace, p. 69 , pl. 13, fig. 8 (Fide Ellis \& Messina 1940)

1950 Eponides ouachitaensis Howe \& Wallace Ruiz de Gaona \& Colom, p. 378, fig. 13/3644

Locality: Vr-004

“Eponides" dalmatinus (de Witt Puyt), 1941

Pl. 11, Figs. 14-15

1941 Eponides carolinensis Cushman var. dalmatina - de Witt Puyt, p. 66, pl. 1, figs. 52 , 57; pl. 2, fig. 2

1950 Eponides carolinensis Cushman var. navarraensis - Ruiz de Gaona \& Colom, p. 377 , fig. $14 / 5-10$

1956 Rotalia dalmatina (de Witt Puyt) - Hagn, p. 165 , pl. 15 , figs. $2-3$

1993 Neoeponides navaransis (Ruiz de Gaona \& Colom) - Sztràkos (In: Mathelin \& Sztràkos), p. 52 , figs. $6-7$

Locality: Vr-005A, Vr-010, Vr-011, Zv/II-

006, Zv/II-010

Family: Mississippinidae Saidova, 1981

Schlosserina asterites (Gümbel), 1868

Pl. 8, Fig. 15

1868 Rosalina asterites - Gümbel, p. 658, pl. 2, fig. $101 \mathrm{a}-\mathrm{c}$

1981 Schlosserina asterites (Gümbel) Lindenberg et al., p. 148

1991 Schlosserina asterites (Gümbel) - Barbin \& Keller-Grünig, p. 241, pl. 1, figs. 13-15

1981 Schlosserina asterites (Gümbel) - Hagn et al., p. 91

1983 Schlosserina asterites (Gümbel) - Setiawan, p. 134 , pl. 15, fig. 1

1993 Schlosserina asterites Hagn - Sztràkos (In: Mathelin \& Sztràkos), p. 80, pl. 15, fig. 2

Locality: Vr-014

Family: Discorbidae Ehrenberg, 1838

Neoeponides schreibersii (d'Orbigny), 1846 
PLATE 9

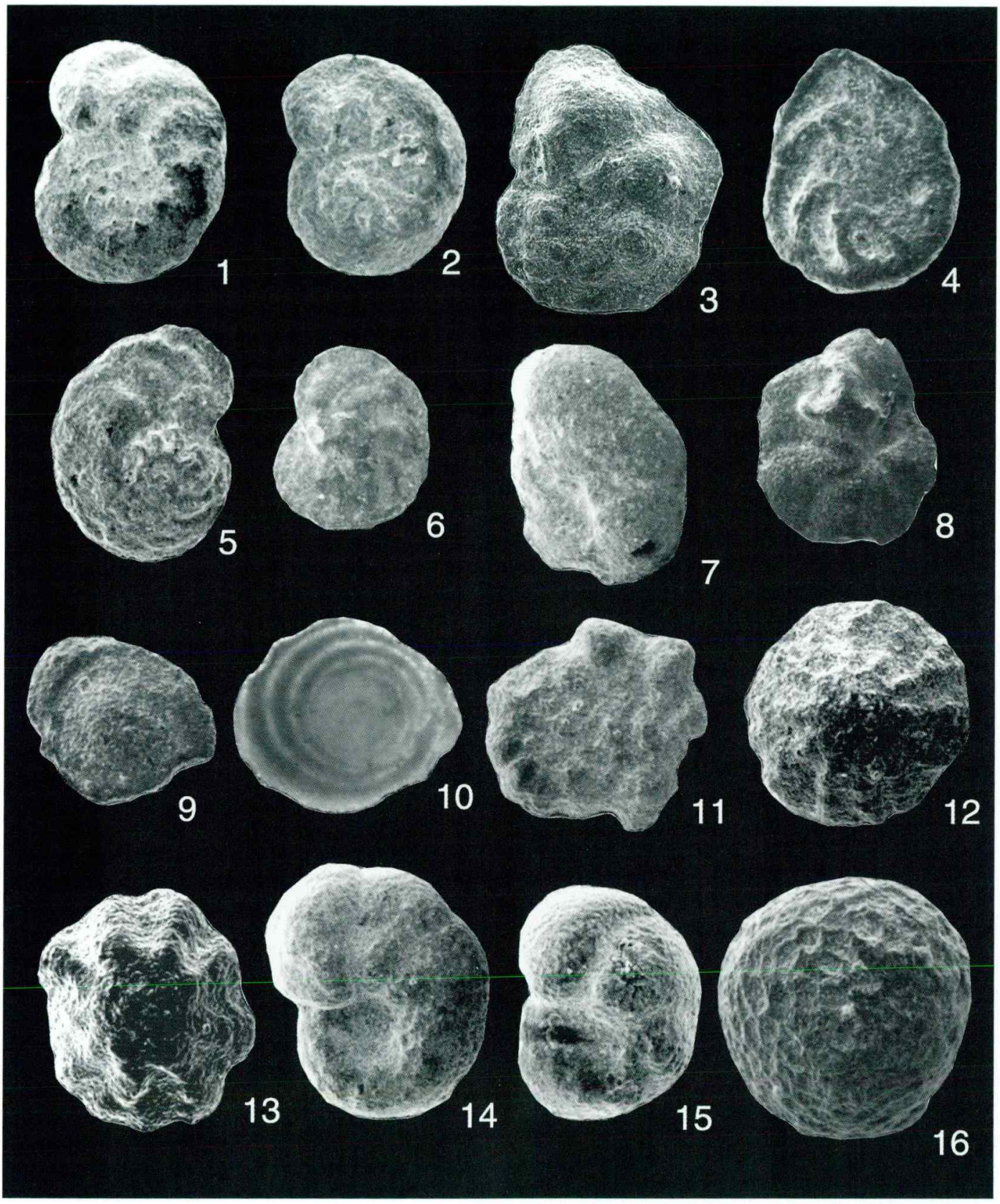

1-2 Cibicidoides ungerianus (d'Orbigny). 1 spiral view, Vr-010, x60. 2 spiral view, Zv/II-008, x60. 3 Laticarinina altocamerata (Heron-Allen \& Earland), umbilical view, Zv/II-008, x80. 4 Planulina compressa (Hantken), Vr-005A, x80. 5-6 Planulina costata (Hantken). 5 spiral view, Vr-012, x40. 6 umbilical view, Vr-010, x40. 7-8 Lobatula lobatula (Walker \& Jacob). 7 umbilical view, Vr-012, x40. 8 umbilical view, Vr-005, x28. 9-10 Cycloloculina eocenica (Terquem). 9 Vr-005A, x80. 10 Vr-005A, x64. 11 Planorbulina difformis Roemer, Vr-005A, x60. 12 Halkyardia minima (Liebus), Vr-011, x80. 13 Halkyardia sp., Vr011A, x120. 14-15 Korobkovella grosserugosa (Gümbel). 14 Vr-010, x60. 15 Vr-010, x60. 16 Sphaerogypsina globula (Reuss), Vr-005A, x120. 
Pl. 8, Figs. 11-12

1846 Rotalina schreibersii - d'Orbigny, p. 154, pl. 8, figs. $4-6$

1993 Neoeponides schreibersii (d'Orbigny) Sztràkos (In: Mathelin \& Sztràkos), p. 81, pl. 15 , fig. 8

Locality: Zv-005, Zv-006, Zv-009, Zv-010

Family: Rosalinidae Reiss, 1963

Neoconorbina sp. 1

Pl. 8, Figs. 16-17

Locality: Vr-005, Vr-005A

Family: Parrelloididae Hofker, 1956

Cibicidoides ungerianus (d'Orbigny), 1846 Pl. 9, Figs. 1-2

1846 Rotalina ungeriana - d'Orbigny, p. 157, pl. 8, figs. 16-18

1950 Cibicides granosus (Reuss) - Ruiz de Gaona \& Colom, p. 368, fig. 13/21-27

1956 Cibicides ungerianus (d'Orbigny) - Hagn, p. 181, pl. 17, figs. 10-11

1975 Heterolepa ungeriana (d'Orbigny) - Braga et al., p. 98

1991 Cibicidoides ungerianus (d'Orbigny) Barbin \& Keller-Grünig, p. 24

1993 Cibicidoides ungerianus (d'Orbigny) Sztràkos (In: Mathelin \& Sztràkos), p. 81, pl. 16, fig. 11

Locality: Vr-004, Vr-010, Vr-011, Zv-004,

$\mathrm{Zv}-006, \mathrm{Zv}_{\mathrm{V}} 008$

Family: Discorbinellidae Sigal, 1952

\section{Laticarinina altocamerata (Heron-Allen \& Earland), 1922}

Pl. 9, Fig. 3

1922 Truncatulina tenuimargo Brady var. altocamerata - Heron-Allen \& Earland, p. 209, pl. 7, figs. 24-27 (Fide Ellis \& Messina 1940)

1964 Laticarinina altocamerata (Heron-Allen \& Earland) - Loeblich \& Tappan, p. C580, fig. 457, 4

1983 Laticarinina altocamerata (Heron-Allen \& Earland) - Setiawan, p. 116. pl. 5, fig. 4

1991 Laticarinina altocamerata (Heron-Allen \& Earland) - Barbin \& Keller-Grünig, p. 242

Locality: Vr-005A, Zv-008

Family: Planulinidae Bermudez, 1952

\section{Planulina compressa (Hantken), 1875}

Pl. 9, Fig. 4

1875 Truncatulina compressa - Hantken, p. 72, pl. 8 , fig. $8 \mathrm{a}-\mathrm{b}$
1956 Planulina compressa (Hantken) - Hagn, p. 179 , pl. 17 , fig. $5 \mathrm{a}-\mathrm{b}$

1975 Planulina compressa (Hantken) - Braga et al., p. 95, pl. 2, fig. 13a-c

1993 Planulina compressa (Hantken) - Sztràkos (In: Mathelin \& Sztràkos), p. 81, pl. 17, fig. 1

Locality: Vr-005A

\section{Planulina costata (Hantken), 1875}

Pl. 9, Figs. 5-6

1875 Truncatulina costata-Hantken, p. 73, pl. 9, fig. 2

1956 Planulina costata Hantken - Hagn, p. 178, pl. 17, figs. 3a-b, 4a-b

1984 Planulina costata (Hantken) - Grünig \& Herb, p. 262

1993 Planulina costata (Hantken) - Sztràkos (In: Mathelin \& Sztràkos), p. 81, pl. 16, fig. 13; pl. 38, fig. 9

Locality: Vr-004, Vr-005, Vr-005A, Vr-

010, Vr-011, Vr-012, Zv-005, Zv-006, Zv-

007, Zv-008, Zv-009, Zv-010

Family: Cibicididae Cushman, 1927

Lobatula lobatula (Walker \& Jacob), 1798

Pl. 9, Figs. 7-8

1798 Nautilus lobatulus - Walker \& Jacob (in Kanmacher), p. 642, pl. 14, fig. 36 (Fide Ellis \& Messina 1940))

1957 Cibicides lobatulus (Walker \& Jacob) - Sacal \& Debourle, p. 68, pl. 33, fig. 1

1962 Cibicides lobatulus (Walker \& Jacob) Gohrbandt, p. 113

1991 Cibicides lobatulus (Walker \& Jacob) Barbin \& Keller-Grünig, p. 240, pl. 1, figs. 17-18

1993 Lobatula lobatula (Walker \& Jacob) Sztràkos (In: Mathelin \& Sztràkos), p. 81, pl. 17 , fig. 9

Locality: Vr-005, Vr-005A, Zv-005, Zv010

Cycloloculina eocenica (Terquem), 1882

Pl. 9, Figs. 9-10

1882 Planorbulina eocenica - Terquem, p. 90, pl. 9 , fig. $15 \mathrm{a}-\mathrm{b}$

1970 Cycloloculina eocenica (Terquem) - Le Calvez, p. 186, pl. 35, fig. 4

1993 Cycloloculina eocenica (Terquem) Sztràkos (In: Mathelin \& Sztràkos), p. 81, pl. 19, fig. 1 ; pl. 43 , fig. 6

Locality: Vr-004, Vr-005A

Family: Planorbulinidae Schwager, 1877

Planorbulina difformis Roemer, 1838

Pl. 9, Fig. 11 


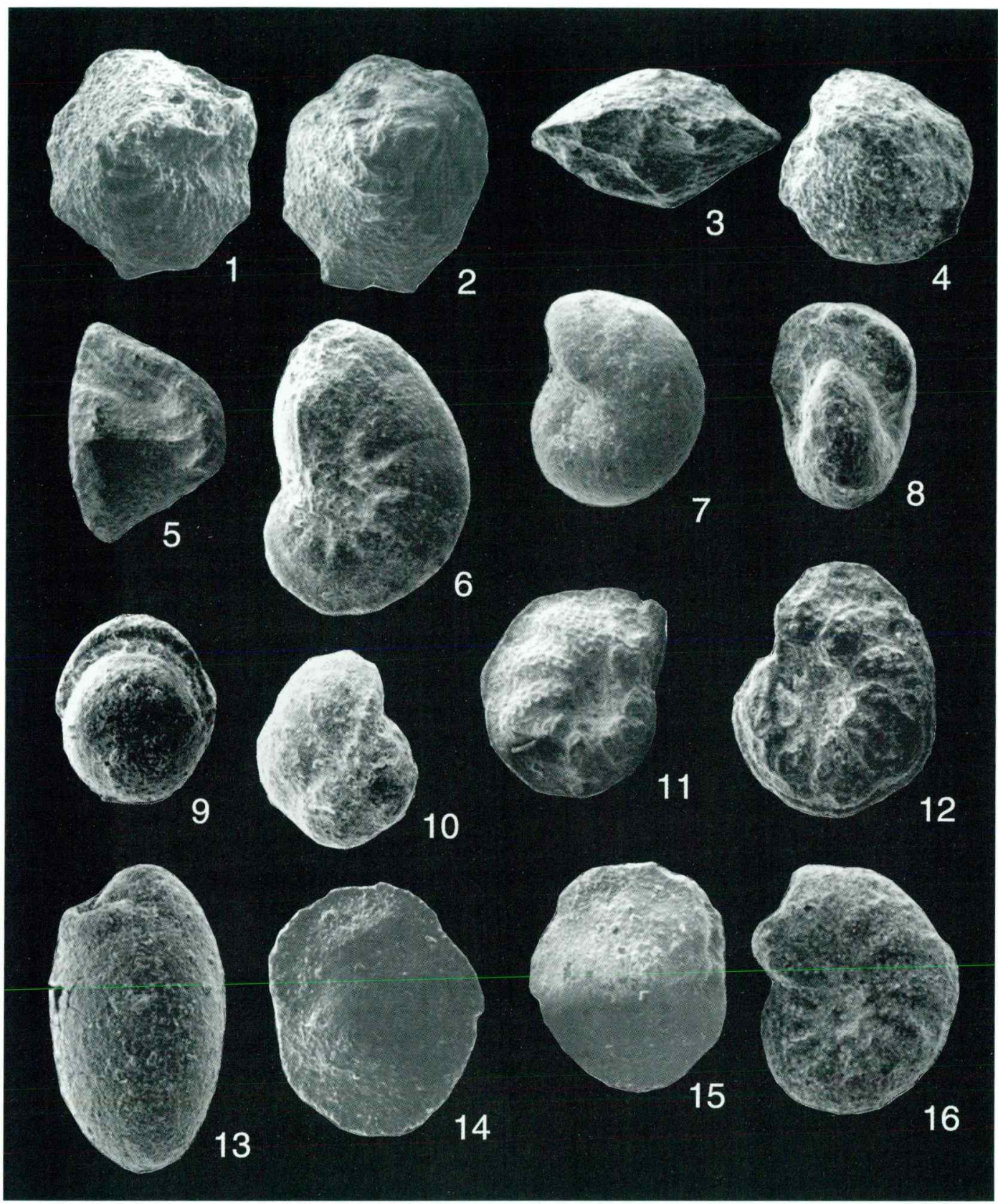

1-2 Asterigerina brencei Haque. 1 umbilical view, Vr-005A, x120. 2 umbilical view, Vr-005A, x120. 34 Asterigerina sp. 3 edge view, Vr-005, x80. 4 umbilical view, Vr-004, x80. 5 Asterigerina rotula (Kaufmann), edge view, Vr-009, x40.6 Nonion scaphum (Fichtel \& Moll), side view, Vr-005, x80. 7-8 Melonis halkyardi (Cushman). 7 side view, Zv/II-008, x80. 8 apertural view, Vr-005, x80.9 Pullenia bulloides (d'Orbigny), apertural view, Zv/II-011, x80. 10 Pullenia quinqueloba (Reuss), side view, Vr-010, x80.11-12 Queraltina epistominoides Marie. $11 \mathrm{Vr}-005 \mathrm{~A}, \mathrm{x} 60.12$ Marie, Vr-005A, x60. 13 Chilostomella ovoidea Reuss, Zv/II-011, x60. 14-15 Charltonina budensis (Hantken). 14 spiral view, Vr-005A, x60. 15 umbilical view, Vr-005A, x60.16 Anomalinoides affinis (Hantken), Vr-005, x80. 
1993 Planorbulina difformis Roemer - Sztràkos (In: Mathelin \& Sztràkos), p. 81, pl. 38, figs. 5-6

Locality: Vr-005A

Family: Cymbaloporidae Cushman, 1927

Halkyardia minima (Liebus), 1911

Pl. 9, Fig. 12

1911 Cymbalopora radiata Hagenow var. minima - Liebus, p. 952, pl. 3, fig. 7a-c

1969 Halkyardia minima (Liebus) - Cimerman, p. 298, pl. 58, figs. 1-6

1976 Halkyardia minima (Liebus) - Šikić et al., p. 195 , pl. 6 , fig. 1

1979 Halkyardia minima (Liebus) - Drobne et al., p. 158

1985 Halkyardia minima (Liebus) - Grünig, p. 272 , pl. 9, figs. 16-17

1991 Halkyardia minima (Liebus) - Barbin \& Keller-Grünig, p. 241

1993 Halkyardia minima (Liebus) - Sztràkos (In: Mathelin \& Sztràkos), p. 54, pl. 38, fig. 12 Locality: Vr-005A, Vr-011, Vr-011A, Vr014

\section{Halkyardia sp.}

Pl. 9, Fig. 13

Locality: Vr-011A

Family: Stilostomellidae Finlay, 1947

Nodogenerina atlantisae (Cushman), 1939

Pl. 8, Figs. 5-6

1939 Ellipsonodosaria atlantisae - Cushman, p. 70, pl. 12, figs. 3-4 (Fide Ellis \& Messina 1940)

1993 Nodogenerina atlantisae (Cushman) Sztràkos (In: Mathelin \& Sztràkos), p. 80, pl. 13, figs. 16-18; pl. 34, figs. 18-19

Locality: Zv-006, Zv-008

\section{Family: Victoriellidae Chapman \&}

Crespin, 1930

Korobkovella grosserugosa (Gümbel), 1868 Pl. 9, Figs. 14-15

1868 Truncatulina grosserugosa - Gümbel, p. 82, pl. 2, fig. 104

1950 Anomalina grosserugosa (Gümbel) - Ruiz de Gaona \& Colom, p. 362

1957 Anomalina grosserugosa (Gümbel) - Sacal \& Debourle, p. 66, pl. 33, fig. 6

1970 Anomalina grosserugosa (Gümbel) - Le Calvez, p. 198, pl. 40, fig. 2

1983 Anomalina grosserugosa (Gümbel) Setiawan, p. 132, pl. 14, fig. 1
1993 Korobkovella grosserugosa (Gümbel) Sztràkos (In: Mathelin \& Sztràkos), p. 82, pl. 19. Fig. 2

Locality: Vr-004, Vr-005A, Vr-009, Vr-

010, Vr-012, Vr-014, Zv-006, Zv-007

\section{Family: Acervulinidae Schultze, 1854}

\section{Sphaerogypsina globula (Reuss), 1848}

Pl. 9, Fig. 16

1848 Ceriopora globulus - Reuss, p. 33, pl. 5, fig. 7

1950 Gypsina globula (Reuss) - Ruiz de Gaona \& Colom, p. 380

1979 Sphaerogypsina globula (Reuss) - Drobne et al., p. 158, pl. 3, fig. 1; pl. 4, figs. 3-4

1985 Sphaerogypsina globula (Reuss) - Grünig, p. 272, pl. 9, figs. 14-15

1993 Sphaerogypsina globula (Reuss) - Sztràkos (In: Mathelin \& Sztràkos), p. 82, pl. 38, fig. 13

Locality: Vr-004, Vr-005, Vr-005A

Family: Asterigerinidae d'Orbigny, 1839

Asterigerina brencei Haque, 1960

Pl. 10, Figs. 1-2

1960 Asterigerina brencei Haque - Setiawan, p. 118, pl. 9, fig. 3

Locality: Vr-005, Vr-009, Vr-010

Asterigerina rotula (Kaufmann), 1867

Pl. 10, Fig. 5

1867 Hemistegina rotula - Kaufmann, p. 150, pl. 8, fig. 19 (Fide Ellis \& Messina 1940)

1886 Pulvinulina rotula (Kaufmann) - Uhlig, p. 193, pl. 3, figs. 5-6; pl. 5, figs. 6-7

1979 Asterigerina rotula (Kaufmann) - Drobne et al., p. 158 , pl. 3, fig. 2 ; pl. 4, fig. 5

1993 Asterigerina rotula (Kaufmann) - Sztràkos (In: Mathelin \& Sztràkos), p. 82, pl. 19, fig. 7

Locality: Vr-010, Vr-011

Asterigerina sp. 1

Pl. 10, Figs. 3-4

Locality: Vr-005, Vr-005A

Family: Nonionidae Schultze, 1854

Nonion scaphum (Fichtel et Moll), 1798 Pl. 10, Fig. 6

1798 Nautilus scapha - Fichtel \& Moll, p. 105, pl. 19, figs. d-f (Fide Ellis \& Messina 1940)

1846 Nonionina communis d'Orbigny d'Orbigny, p. 106, pl. 5, fig. 7 


\section{PLATE 11}

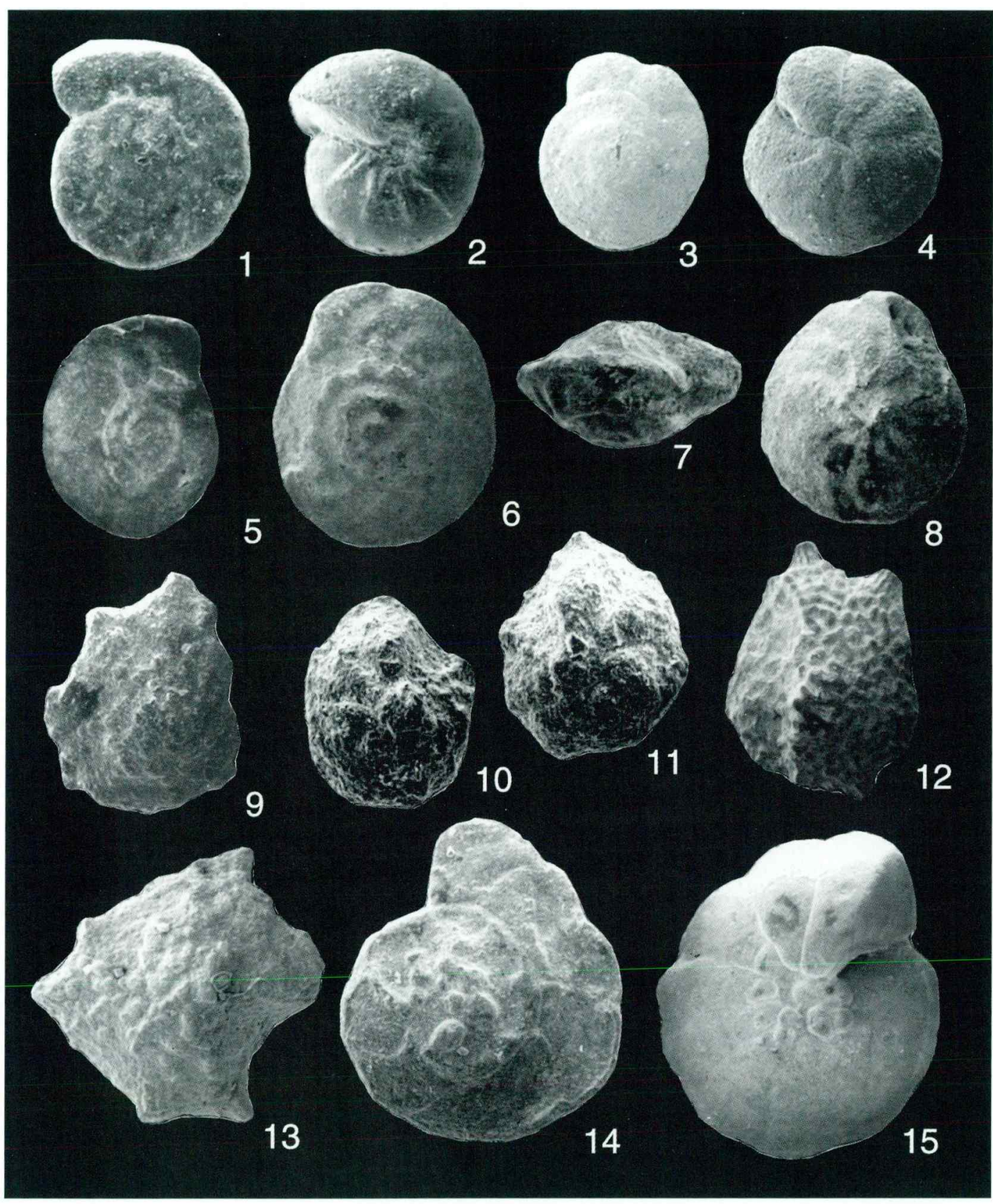

1-2 Gyroidinoides girardanus (Reuss). 1 spiral view, Zv/II-006, x40. 2 umbilical view, Zv/II-006, x40. 34 Heterolepa eocaena (Gümbel). 3 spiral view, Zv/II-006, x28. 4 umbilical view, Zv/II-011, x35. 5 Heterolepa reussi (Silvestri), spiral view, Vr-012, x50. 6-8 Gavelinella acuta (Plummer). 6 spiral view, Vr-009, x40. 7 edge view, Vr-009, x400.8 spiral view, Vr-009, x40.9 Pararotalia armata (d'Orbigny), spiral view, Vr011, x60. 10-11 Pararotalia audouini (d'Orbigny). 10 spiral view, Vr-011, x80. 11 spiral view, Vr-011, x80. 12-13 Calcarina lecalvezae Boulanger \& Poignant. 12 Vr-014, x28. 13 Zv/II-009, x28. 14-15 "Eponides" dalmatinus (de Witt Puyt). 14 spiral view, Vr-009, x28, 15 umbilical view, Zv/II-010, x28. 
1961 Nonion scaphum (Fichtel \& Moll) Kaasschieter, p. 204, pl. 10, fig. 5

1993 Nonion commune (d'Orbigny) - Sztràkos (In: Mathelin \& Sztràkos), p. 82, pl. 19, fig. 11

Locality: Vr-005

\section{Melonis halkyardi (Cushman), 1936}

Pl. 10, Figs. 7-8

1936 Nonion halkyardi - Cushman, p. 63, pl. 12, fig. 1 (Fide Ellis \& Messina 1940)

1987a Melonis halkyardi (Cushman) - Sztràkos, p. 32 , pl. 15 , fig. 6

Locality: Vr-005, Vr-012, Zv/II-008, Zv/II011

\section{Pullenia bulloides (d'Orbigny), 1846}

Pl. 10, Fig. 9

1846 Nonionina bulloides - d'Orbigny, p. 107, pl. 5, fig. 9-10

1875 Pullenia bulloides (d'Orbigny) - Hantken, p. 59, pl. 10, fig. 9

1987a Pullenia bulloides (d'Orbigny) - Sztràkos, p. 34

Locality: Vr-005A, Vr-011, Vr-011A, Zv-

006, Zv-010, Zv-011

\section{Pullenia quinqueloba (Reuss), 1851}

Pl. 10, Fig. 10

1851 Nonionina quinqueloba - Reuss, p. 71, pl. 5, fig. 31

1987 Pullenia quinqueloba (Reuss) - Wenger, p. 299, pl. 13, figs. 16, 20

1991 Pullenia quinqueloba (Reuss) - Barbin \& Keller-Grünig, p. 240

1993 Pullenia quinqueloba (Reuss) - Sztràkos (In: Mathelin \& Sztràkos), p. 82, pl. 19, fig. 17

Locality: Vr-005A, Zv-005, Zv-006

Family: Almaenidae Myatlyuk, 1959

\section{Queraltina epistominoides Marie, 1950}

Pl. 10, Figs. 11-12

1950 Queraltina epistominoides - Marie, p. 74, figs. 1-3, 8-9 (Fide Ellis \& Messina 1940)

1983 Queraltina epistominoides Marie Setiawan, p. 133, pl. 14, figs. 2-3

1985 Almaena epistominoides (Marie) - Grünig, p. 276

1987a Queraltina epistominoides Marie - Sztràkos, p. 34

1991 Queraltina epistominoides Marie - Barbin \& Keller-Grünig, p. 241, pl. 2, fig. 16

1993 Queraltina epistominoides Marie - Sztràkos (In: Mathelin \& Sztràkos), p. 56, pl. 39, fig. 20

Locality: Vr-004, Vr-005A, Vr-012, Vr-014

\section{Family: Chilostomellidae Brady, 1881}

\section{Chilostomella ovoidea Reuss, 1850}

Pl. 10, Fig. 13

1850 Chilostomella ovoidea - Reuss, p. 380, pl. 48, fig. 12

1993 Chilostomella cf. ovoidea Reuss - Sztràkos (In: Mathelin \& Sztràkos), p. 82, pl. 20. fig. 1

Locality: Zv-005, Zv-006, Zv-009, Zv-010, $\mathrm{Zv}-011$

Family: Osangulariidae Loeblich \&

Tappan, 1964

Charltonina budensis (Hantken), 1875

Pl. 10, Figs. 14-15

1875 Truncatulina budensis - Hantken, p. 75, pl. 8, fig. 6

1919 Pulvinulina acutimargo - Halkyard, p. 120, pl. 7 , fig. 4

1985 Alabamina acutimargo (Halkyard) - Grünig, p. 274 , pl. 10. fig. 9-11

1987aAlabamina budensis (Hantken) - Sztràkos, p. 32 , pl. 12 , fig. 8

1993 Charltonina budensis (Hantken) - Sztràkos (In: Mathelin \& Sztràkos), p. 57, pl. 20, fig. 4

Locality: Vr-005A, Vr-009, Vr-010, Vr-

011, Vr-012

Family: Heterolepidae Gonzales-Donoso, 1969

\section{Anomalinoides affinis (Hantken), 1875}

Pl. 10, Fig. 16

1875 Pulvinulina affinis - Hantken, p. 78, pl. 10, fig. 6

1941 Anomalina dalmatina nov. sp. - van Bellen, p. 1001, fig. 26a-c

1956 Anomalinoides dalmatinus (van Bellen) Hagn, p. 177, pl. 17, fig. 1a-b

1993 Anomalinoides affinis (Hantken) - Sztràkos (In: Mathelin \& Sztràkos), p. 82, pl. 20, fig. 8

Locality: Vr-004, Vr-005A, Zv-006, Zv-

007, Zv-009, Zv-010, Zv-011

\section{Heterolepa eocaena (Gümbel), 1868}

Pl. 11, Fig. 3-4

1868 Rotalia eocaena - Gümbel, p. 72, pl. 2, fig. $87 \mathrm{a}-\mathrm{b}$

1956 Cibicides eocaenus (Gümbel) - Hagn, p. 180, pl. 17, figs. $8-9$

1985 Heterolepa eocaena (Gümbel) - Grünig, p. 275, pl. 11, figs. 1-3

Locality: Vr-004, Vr-005, Vr-005A, Vr- 
PLATE 12

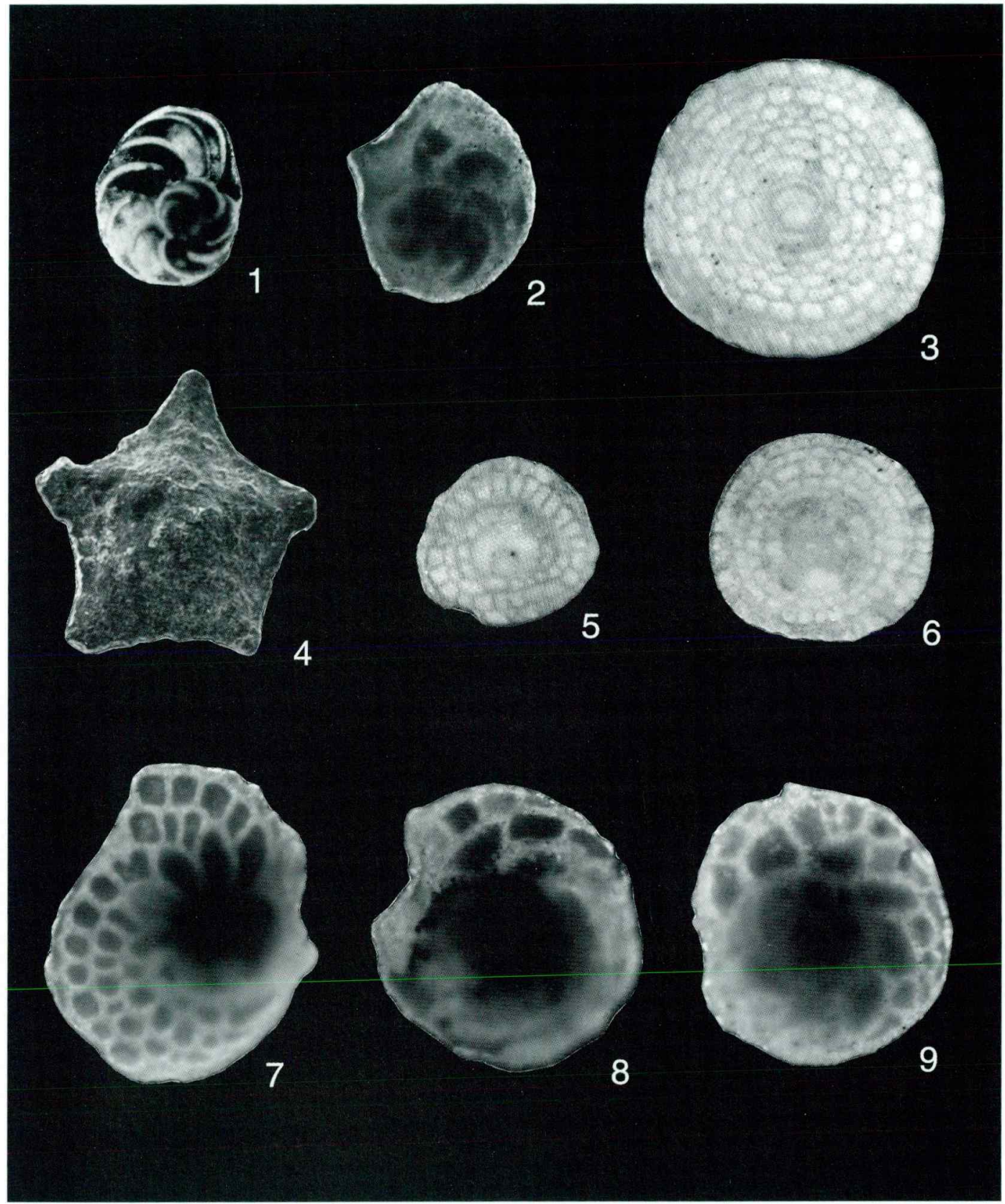

1-2 Operculina cf. gomezi Colom \& Bauza. 1 Vr-005A, x32. 2 Vr-005A, x32. 3 Eoannularia eoceniva Cole \& Bermudez, Vr-005A, x64. 4 Asterocyclina sp. Vr-005A, x80. 5 Discocyclina trabayensis vicenzensis Less, Vr-005A, x64. 6 Discocyclina s. 1., Vr-005A, x64. 7-9Heterostegoina sp. 1. 7 Vr-005A, x64 . 8 Vr005A, x64. 9 Vr-005A, x64. 
012, Zv-005, Zv-011

Heterolepa reussi (Silvestri), 1906

Pl. 11, Fig. 5

1906 Truncatulina dutemplei (d'Orbigny) var. reussi - Silvestri, p. 33 (Fide Ellis \& Messina 1940)

1956 Cibicides dalmatinus van Bellen - Hagn, p. 180 , pl. 18, fig. 5a-b

1985 Heterolepa reussi (Silvestri) - Grünig, p. 76, pl. 11, figs. 7-9

Locality: Vr-011, Vr-012

Family: Gavelinellidae Hofker, 1956

Gyroidinoides girardanus (Reuss), 1851

Pl. 11, Figs. 1-2

1851 Rotalina girardana - Reuss, p. 73, pl. 5, fig. 34

1993 Gyroidinoides girardanus (Reuss) - Sztràkos (In: Mathelin \& Sztràkos), p. 83, pl. 21, fig. 3

Locality: Vr-004, Vr-005A, Vr-010, Vr-

012, Zv-006, Zv-007, Zv-009, Zv-011

Gavelinella acuta (Plummer), 1926

Pl. 11, Figs. 6-8

1926 Anomalina ammonoides (Reuss) var. acuta n. var. - Plummer, p. 149, pl. 10, fig. 2a-c

1983 Anomalina acuta Plummer - Setiawan, p. 131, pl. 14, fig. 3

1987a Gavelinella acuta (Plummer) - Sztràkos, p. 31 , pl. 13, figs. 12,13

Locality: Vr-009

Family: Linderinidae Loeblich \& Tappan, 1984

\section{Eoannularia eocenica Cole \& Bermudez, 1944}

Pl. 12, Fig. 3

1944 Eoannularia eocenica - Cole \& Bermudez, p. 12, pl. 1, figs. 11-16 (Fide Ellis \& Messina 1940)

1976 Eoannularia eocenica Cole \& Bermudez Sirel, p. 80 , pl. 1. figs. 1-3; pl. 2, figs. 1, 2, 4, 5, 8, 9

Locality: Vr-005A, Vr-011

Family: Rotaliidae Ehrenberg, 1839

Pararotalia armata (d'Orbigny), 1826

Pl. 11, Fig. 9

1826 Rotalia armata - d'Orbigny, p. 273, pl. 8, fig. no. 22
1970 Pararotalia armata (d'Orbigny) - Le Calvez, p. 161 , pl. 39 , fig. 1

1985 Pararotalia armata (d'Orbigny) - Grünig, p. 270, pl. 8, fig. 9

1993 Pararotalia armata (d'Orbigny) - Sztràkos (In: Mathelin \& Sztràkos), p. 83, pl. 42, fig. 11

Locality: Vr-011

Pararotalia audouini (d'Orbigny), 1826

Pl. 11, Figs. 10-11

1826 Rotalia audouini - d'Orbigny, p. 273, no. 19

1970 Pararotalia audouini (d'Orbigny) - Le Calvez, p. 162 , pl. 34, fig. 8

1975 Pararotalia audouini (d'Orbigny) - Braga et al., p. 107, pl. 6, figs. 8-9

1983 Pararotalia audouini (d'Orbigny) Setiawan, p. 120

1993 Pararotalia audouini (d'Orbigny) - Sztràkos (In: Mathelin \& Sztràkos), p. 83, pl. 42, fig. 12

Locality: Vr-011

Family: Calcarinidae Schwager, 1876

Calcarina lecalvezae Boulanger \&

Poignant, 1971

Pl. 11, Figs. 12-13

1971 Calcarina le calvezae - Boulanger \& Poignant, p. 96, pl. 1, figs. 1-10

1991 Calcarina lecalvezae Boulanger \& Poignant - Barbin \& Keller-Grünig, p. 242, pl. 1, fig. 22

1993 Calcarina lecalvezae Boulanger \& Poignant - Sztràkos (In: Mathelin \& Sztràkos), p. 83, pl. 22, figs. 7-9

Locality: Vr-014, Zv-009

Family: Nummulitidae de Blainville, 1827

Heterostegina sp. 1

Pl. 12, Figs. 7-9

Locality: Vr-005, Vr-005A

Operculina cf. gomezi Colom \& Bauza, 1950

Pl. 12, Figs. 1-2

1950 Operculina canalifera gomezi - Colom \& Bauza, p. 217, pl. 16, figs. 1-3; texte, figs. 12. (Fide Ellis \& Messina 1940)

1971 Operculina canalifera gomezi Colom \& Bauza - Ferrer, p. 35, fig. 19

1977 Operculina gomezi Colom \& Bauza Hottinger, p. 98, figs. $38 \mathrm{~A}-\mathrm{F}$

1993 Operculina gomezi Colom \& Bauza Mathelin (In: Mathelin \& Sztràkos), p. 62, pl. 44 , fig. 4 
Locality: Vr-005A

Family: Discocyclinidae Galloway, 1928

\section{Discocyclina trabayensis vicenzensis Less, 1987}

Pl. 12, Fig. 5

1987 Discocyclina trabayensis Neumann, 1955 vicenzensis $\mathrm{n}$. ssp. - Less, p. 172, pl. 18, figs. $3-5$, textfig. $27 \mathrm{y}$

Locality: Vr-005A

\section{Discocyclina s. 1 .}

Pl. 12, Fig. 6

Locality: Vr-004, Vr-005, Vr-005A, VR009

\section{Asterocyclina sp. 1}

Pl. 12, Fig. 4

Locality: Vr-004, Vr-005, Vr-005A

\section{Acknowledgemets}

We thank the Slovenian Research Agency for financing this project. To the late Monique Toumarkine, who in the meantime passed away, go our thanks for determinations of planktic species, to Vlasta Cosović for determination of Lepidocyclina, and to Jože Čar, Ladislav Placer and Laszlo Fodor for discussions on tectonic deformations in profiles. Thanks also to Tom Popit and Alenka Eva Črne who technically prepared the plates, and to Simon Pirc for translation of the text into English, and to Glenn S. Jacks for English corrections of the final version.

\section{References}

Aničić, B. \& Juriša, M. 1985a: Tolmač za list Rogatec, Osnovna geološka karta SFRJ 1:100000 (Geology of the Sheet Rogatec, Geological Map of SFRJ 1:100000). - Zvezni geološki zavod, 76 pp., Beograd (in Slovenian with English abstract).

Aničić, B. \& Juriša, M. 1985b: List Rogatec, Osnovna geološka karta SFRJ 1:100000 (Sheet Rogatec, Geological Map of SFRJ 1:100000). Zvezni geološki zavod, Beograd.

B a ld i, T. 1984: The terminal Eocene and Early Oligocene events in Hungary and the separation of an anoxic, cold Paratethys. - Eclogae geol. Helv., 77, 1-27, Basle.

Barbin, V. \& Keller-Grünig, A. 1991: Benthic foraminiferal assebmblages from the Brendola section (Priabonian stage stratotype area, northern Italy): Distribution, palaeoenvironment and palaeoecology. - Marine Micropaleontology, 17, 237-254, Amsterdam.

Barker, R. W. 1960: Taxonomic Notes on the Species Figured by H. B. Brady in his Report on the Foraminifera Dredged by H. M. S. Challenger During the Years 1873-1876. Accompanied by a Reproduction of Brady's Plates: Soc. Econom. Paleont. Miner., Spec. publ. 9, 238 pp., pls. 1-115, Tulsa.

Bellen, van R. C. 1941: Some eocene Foraminifera from the neighbourhood of Ričice near Imotski, E Dalmatia, Yugoslavia. - Proc. Ned. Akad. Wet., 44, 996-1005, Amsterdam.

Bittner, A. 1884: Die Tertiär-Ablagerungen, von Trifail und Sagor. - Jahrb. k.k. Geol. Reichsanst., 34, 433-596, Wien.

B ornemann, P. 1855: Die mikroskopische Fauna des Septarienthones von Hermsdorf bei Berlin. - Zeitschrift Deutsch. Geol. Ges. 7, 307371 , pls. 12-21, Berlin.

Boulanger, D. \& Poignant, A. 1971: Sur l'existence du genre Calcarina en Aquitaine méridionale. - Revue de Micropaléontologie, 14 (2), 96-101, Paris.

Braga, GP., De Biase, R., Grünig, A. \& Proto Decima, F. 1975: Foraminiferi bentonici del Paleocene ed Eocene della Sezione di Possagno. In: Bolli, H. M. (ed.): Monographia micropaleontologica sul Paleocene e l'Eocene di Possagno. Provincia di Treviso, Italia. - Schweiz. paläont. Abh., 97, 85-371, Basel.

Brezigar, A., Ogorelec, B., Rijavec, L. \& Mi o č, P. 1988: Geološka zgradba predpliocenske podlage Velenjske udorine in okolice (Geologic setting of the Pre-Pliocene basement of the Velenje depression and its surroundings ). - Geologija, 30, 31-65, Ljubljana (in Slovenian with English abstract).

Buch, L. 1851: Abhandlungen über die Lagerung der Braunkohlen in Europa. - Berichte k. Akad. Wiss., 683-701, Berlin.

Buser, S. 1979: Tolmač lista Celje, Osnovna geološka karta SFRJ 1:100000 (Geology of the Sheet Celje, Geological Map of SFRJ 1:100000). Zvezni geološki zavod, 72 pp., Beograd (in Slovenian with English abstract).

Cimerman, F. 1969: Halkyardia maxima n. sp. (Middle Oligocene) and Halkyardia minima (Liebus) (Middle Eocene). - Rocz. Polsk. Tow. Geol., 39, 295-304, pls. 57-58, Krakow.

Cimerman, F. 1979: Oligocene beds in Slovenia. - In: K. Drobne (ed.), $16^{\text {th }}$ European Micropaleontological colloquium. - Croatian Geological Society, Slovenian Geological Society, 6570, Ljubljana.

Csontos, L., Nagymarosy, A., Horváth, F.\& Kováč, M. 1992. Tertiary evolution of the intra-Carpathian area: a model. - Tectonophysics, 199, 73-91, Amsterdam.

Cushman, J. A. 1937a: A Monograph of the foraminiferal family Valvulinidae. - Cushman. Lab. Foram. Res. Spec. Publ. 8, 210 pp., pls. 1-24, Sharon Mass.

Cushman, J. A. 1937b: A Monograph of the subfamily Virgulininae of the foraminiferal family Buliminidae. - Cushman. Lab. Foram. Res. Spec. Publ. 9, 228 pp., pls. 1-24, Sharon Mass.

Cuvillier. J. \& Szaka11, V. 1949: Foraminifères deuitaine, Première partie (Reophacidae à Nonionidae). - Soc. Nat. Pétr. Aquit., 113 pp., pls. 1-32, Toulouse. 
Drobne, K., Pavlovec, R. \& Drobne, F.: 1979: Mikrofosilne karakteristike starejšega paleogena na zahodnem obrobju Panonskega bazena (Characteristics of microfossils in the old Paleogene on the western border of the Pannonian basin). - Znanstveni savjet za naftu Jugoslavenske akademije znanosti i umjetnosti, Stubičke Toplice, Zbornik radova, 155-166, Zagreb (in Slovenian with English abstract).

Drobne, K., Pavlovec, R., Drobne, F., Cimerman, F. \& Šikić, L. 1985: Nekatere velike foraminifere iz zgornjeeocenskih in bazalnih oligocenskih skladov v severni Sloveniji (Some larger foraminifera from the Upper Eocene and the basal Oligocene beds in North Slovenia).

Geološki glasnik, 28, 77-117, Sarajevo (in Slovenian with English abstract).

Ellis, B. F. \& Messina, A. R. 1940: Catalogue of Foraminifera. - American Museum of Natural Sciences, New York.

Ettingshausen, C. 1853: Über die fossile Flora des Monte Promina in Dalmatien. Sitzungsberichte k. Akad. Wiss., mathem.naturwiss. Cl., 10, 424-428, Wien.

Ettingshausen, C. 1858: Beiträge zur Kenntniss der fossilen Flora von Sotzka in Untersteiermark. - Sitzungsberichte k. Akad. Wiss., mathem.-naturwiss. Cl., 28/6, 471-567, 6 Taf., Wien.

Ettingshausen, C. 1872: Die fossile Flora von Sagor in Krain, 1. - Denkschriften k. Akad. Wiss. mathem.-naturwiss. Cl. 32, 159-202, 10 Taf., Wien.

Ettingshausen, C. 1877: Die fossile Flora von Sagor in Krain, 2. - Denkschriften K. Akad. Wiss. mathem.-naturwiss. Cl., 37, 161-216, 27 Taf., Wien.

Ettingshausen, C. 1885: Die fossile Flora von Sagor in Krain, 3. - Denkschriften k. Akad. Wiss. mathem.-naturwiss. Cl., 50, 1-54, 5 Taf., Wien.

Ferrer, J. 1971: El Paleoceno y Eoceno del borde sur-oriental de la depresión del Ebro (Cataluna). - Schweiz. Pal. Abh., 90, 1-70, pls. 18, Basel.

Foraminiferi Padani (1982) (Terziario e Quaternario). Atlante iconografico e distribuzione stratigrafica. Sec. edizione, 1982, Agip S. p. A., pls. 1-52, Milano.

Fornasini, C. 1904: Illustrazione di specie orbignyane di foraminiferi istituite nel 1826. Mem. R. Accad.Sci. Ist. Bologna, Ser. 6 (1), 1-17, pls. 1-4, Bologna.

Gibson, T. G., B arbin, V., Poignant, A. \& Sztràkos, K. 1991, Early evolution of Tubulogenerina during the Paleogene of Europe. - J. Foram. Res. 21 (4), 299-312, Washington.

Gohrbandt, K. 1961: Die Kleinforaminiferenfauna des obereozänen Anteils der Reingruber Serie bei Bruderndorf (Bezirk Korneuburg, Niederösterreich). - Mitt.Geol. Ges. Wien, 54, 55145, pls. 1-8, Wien.

Grünig, A. 1985: Systematical description of Eocene benthic foraminifera of Possagno (Northern Italy), Sansoain (Northern Spain) and Biarritz (Aquitaine, France). - Memorie Sci. Geol. Univ. Padova, 37, 251-302, Padova.

Grünig, A. \& Herb, R. 1984: Eocene Benthic Foraminifera from Biarritz: Distribution and Paleoenvironment. - Benthos '83, $2^{\text {nd }}$ Internat Symp. Benthic Foraminifera, 257-265, Pau-Bordeaux.
Gümbel, C. W. 1868: Beiträge zur Foraminiferenfauna der nordalpinen, älteren Eozägebilde oder der Kressenberger Nummulitenschichten. - Abhandungen k. bayer. Akad. Wiss. II. Cl., 10, II. Abth, 1-152, pls. 1-4, München.

Hagn, H. 1956: Geologische und paläontologische Untersuchungen im Tertiär des Monte Brione und seiner Umgebung. Palaeontographica, 107, 67-210, pls. 7-18, Stuttgart.

Halkyard, E. 1919: The fossil Foraminifera of the Blue Marl of the Côte des Basques, Biarritz. - Mem. Proc. Manch. Phil. Soc., 62, 1.145, I-XXIV, pls. 1-9, Manchester.

Hamrla, M. 1987: Prispevek h geologiji premogišč zreškega okoliša in uvrstitev njegovih premogov po odsevnosti (Contribution to the geology of coal deposits in the Zreče area and reflectance-based ranking of its coal. - Geologija, 30, 343-390, Ljubljana ( in Slovenian with English summary).

Hantken, M. 1868: A kis-czelli tàlyag foraminiferái. - Mgyarh. Földt. Tàrs. Munk. 4, 75-96, pls. 1-2, Budapest.

Hantken, M. In Posewitz, T. 1889: Borneo. Entdeckungsreisen und Untersuchungen. Gegenwärtiger Stand der geologischen Kenntnisse. Verbreitung der nutzbaren Mnineralien, Berlin.

Hantken, M. 1875: Die Fauna der Clavulina Szaboi Schichten, I. Theil: Foraminiferen. - Mitt. Jb. k. ungar. Geol. Anst., 4, 1-93, pls. 1-16 Budapest.

Hoernes, 1876: Anthracotherium magnum Cuv. Aus den Kohlen-Ablagerungen von Trifail. Jahrb. k.k. Geol. Reichsanst., 26/3, 209-242, Wien.

Hoernes, 1892: Die Kohlenablagerungen von Radeldorf, Stranitzen und Lubnitzengraben bei Rötschach und von St. Britz bei Wollan in Untersteiermark. - Mittheilungen nat.-wiss. Ver. Steiermark, 29, 275-295, Graz.

Hottinger, L. 1977: Foraminifères operculiuniformes. - Mémoires, Sér. C, v. 40, Museum National d'Histoire Naturelle, 1-159, pls. 1-66, Paris.

Jelen, B., Mervič, H., Horvat, A. \& Pavšič, J. 1990: Spremembe mikrofosilnih združb v oligocenu Zasavja (Changes of the microfossil assemblages in the Oligocene of the Zasavje region). - Geologija, 31/ 32, 329-345, Ljubljana (in Slovenian with English abstract).

Jelen, B., Aničič, B., Brezigar, A., Buser, S., Cimerman, F., Drobne, K., Monostori, M., Kedves, M., Pavlovec, R., Pavšič, J. \& Skaberne, D. 1992: Model of positional relationships for Upper Paleogene and Miocene strata in Slovenia. In: A. Montanari, R. Coccioni, G. S. Odin (eds.), Interdisciplinary Geological Conference on the Miocene Epoch with Emphases on the Umbria - Marche - Sequence. Abstracts and field trips, 71-72, International Union of geological Sciences, Subcomission on Geochronology. Ancona, Italy.

Jelen, B., Brezigar, A., Buser, S., Cimerman, F. Drobne, K., Monostori, M., Kedves, M., Pavlovec, R., Pavšič, J. \& Skaberne, D. 1993a: Novo v reševanju problema stratigrafije oligocena in spodnjega miocena v Sloveniji. - Rudarsko-metalurški zbornik, 33. Skok čez kožo, 16-18, Ljubljana.

Jelen, B., Šimunić, A., Drobne, K., Skaberne, D., Ćosović, V., Avanić,R., Báldi- 
Beke, M., Cimerman, F., Čar, J., Fodor, L., Kedves, M., Márton, E., Monostori, M., Pavlovec, R., Placer, L., Śikić, L., Toumarkine, M., Turnšek, D \& Zagoršek, K. 2000: Eocene in NE Slovenia and NW Croatia. $-5^{\text {th }}$ Meeting of the IUGS-UNESCO, IGCP $393-$ July $18^{\text {th }}-31^{\text {st }}, 2000$. Field trip Guidebook, 97147, Bologna.

K a a s s chieter, J. P. H. 1961: Foraminifera of the Eocene of Belgium. - Mémoire Inst. Roy. Sci. Nat. Bel., 147, 1-271, 16 Pl., 8 Tab., Bruxelles.

Kuščer, D. 1955: Nova opazovanja o savski fazi (Neue Beobachtungen über die Sava-Phase). - Geologija, 3, 260-264, Ljubljana (in Slovenian and German).

Kuščer, D. 1967: Zagorski terciar (Tertiary Formations of Zagorje). - Geologija, 10, 5-85, Ljubljana (in Slovenian with English summary).

Le Calvez, Y. 1970: Contribution à l'étude des foraminifères paléogènes du Bassin de Paris. - Cahiers de paléontologie, 1-326, pls. 1-48, Paris.

Les s, G. 1987: Paleontology and Stratigraphy of the European Orthophragminae. - Geologica Hungarica, Ser. Palaeontologica, 51, 1-373, pls. 145, Budapest.

Liebus, A.1911: Die Foraminiferenfauna der mittelocänen Mergel von Norddalmatien. Sitzungsber. K. Akad. Wiss. math.-naturw. Kl., $120,1-92$, pls. $1-3$, Wien

Lindenberg, H. G., Martini, E. \& Witt, W. 1981: C5: Kaiserwald-Graben. In: Hagn, H. (Ed.), Die Bayerischen Alpen und ihr Vorland in mikropaläontologischer Sicht. - Geologica Bavarica, 82, 1-408, München.

Loeblich, A. R. \& Tappan, H. 1964: Sarcodina chiefly "thecamoebians" and Foraminiferida. In: R. C. Moore (ed.) - Treatise on invertebrate Paleontology. Part C, Protista 2, Geological Society of America, Univ. Kansas Press, 1-900.

Loeblich, A. R. \& Tappan, H. 1987: Foraminiferal Genera and their Classification: Van Nostrand Reinhold, 2 vols, 970 pp., pls.1-847, New York.

Majzon, 1958: A Jugoszláviai "Sotzka" rétegek kora. - Földtani Közlöny, 88/3, 354-355, Budapest.

Marie, P. 1950: Queraltina, nouveau genre de Foraminifère de l'Éocene pyrénéen. - Bull. Soc. Gëol. France, ser. 5, 20, 73-80, Paris.

Mathelin, J-C. \& Sztràkos, K. 1993: L'Eocène de Biarritz (Pyrénées Atlantiques, SW France). Stratigraphie et paléoenvironnement. Monographie des Foraminifères. - Cahiers de Micropaléontologie, 8, 5-85, pls. 1-48, Paris.

Mihajlović, Dj. \& Jungwirth, E. 1988: Oligocene aged flora from Novi Dol (Slovenia, Yugoslavia). - Rev. Paléobiologie, 7/2, 435-447, Genève.

Mikuž, V. 1979: Srednjeeocenski moluski iz Lepene (Middle Eocene molluscan fauna from Lepena). - Geologija, 22/2, 189-224, Ljubljana (in Slovenian with English abstract).

Mi oč , P. 1978: Tolmač za list Slovenj Gradec, Osnovna geološka karta SFRJ 1:100000 (Geology of the Sheet Slovenj Gradec, Geological Map of SFRJ 1:100000). - Zvezni geološki zavod, 74 pp., Beograd (in Slovenian with English abstract).

Mi oč, P. \& Žnidarč ič, M. 1977: List Slovenj Gradec, Osnovna geološka karta SFRJ 1:100000 (Sheet Slovenj Gradec, Geological Map of SFRJ 1:100000). - Zvezni geološki zavod, Beograd.

Morlot, A. 1849a: Uebersicht der geologischen Verhältnisse des südlich von der
Drau gelegenen Theiles von Steiermark. - Berichte über die Mittheilungen von Freunden der Naturwissenschaften, 5/3, 174-183, Wien.

Morlot, A. 1849b: Mittheilungen über die geologschen Verältnisse des südlich von der Drau gelegenen Theiles von Steiermark. - Berichte über die Mittheilungen von Freunden der Naturwissenschaften, 5/1, 100, Wien.

Morlot, A. 1850: Andeutungen über die geologischen Verhältnisse des südlichsten Theiles von Untersteyer. - Berichte über die Mittheilungen von Freunden der Naturwissenschaften, 6/9, 159168, Wien.

Morlot, A. 1853: Einige Bemerkungen über die geologischen Verhältnisse von Untersteier. Zweiter Bericht des geognostisch-montanistischen Vereines für Steiermark, Gratz.

Murray, J. W. 1991: Ecology and Palaeoecology of Benthic Foraminifera. Longman Scientific \& Technical, 397 pp., Essex.

Orbigny, A. d' 1826: Tableau méthodique de la classe des Céphalopodes. - Ann. Sci. nat. Ser. 7, 245-316, pls. 10-17, Paris.

Orbigny, A. d' 1846: Foraminifères fossiles du Bassin tertiaire de Vienne (Autriche). - Gide et Comp., 312 pp., pls. 1-21, Paris.

Papp, A. 1954: Miogypsinidae aus dem Oligozän von Zagorje (Slovenian, Jugoslavien) (Miogypsinidae iz Oligocena v Zagorju). Geologija, 2, 168-178, Ljubljana (in German and Slovenian).

Papp, A. 1955: Lepidocyclinen aus Zagorje und Tuhinjska dolina östlich von Kamnik (Slowenien) (Lepidocikline iz Zagorja in Tuhinjske doline). - Geologija, 3, 209-215, Ljubljana (in German and Slovenian).

Papp, A. 1975: Die Grossforaminiferen des Egerien. In: T. Báldi \& J. Seneš (eds), Chronostratigraphie und Neostratotypen, Miozän der Zentralen Paratethys, 5, OM Egerien. - VEDA, 289-307, Bratislava.

Papp.A. \& Schmid, M. E. 1985: Die Fossilen Foraminiferen des tertiären Beckens von Wien. Revision der Monographie von Alcide d'Orbigny (1846). - Abh. Geol. Bundesanstalt Wien, 73, 1311 , pls. $1-102$, Wien.

Pavlovec, R., Drobne, K. \& Drobne, F. 1990: Ostanki eocenskih kamnin na slovenjebistriškem prostoru [Reste von Eozän-Gesteinen in Raum von Slovenska Bistrica]. - Zbornik občine Slovenska Bistrica, 2, 440-448, pls. 1-2, Slovenska Bistrica.

Petrascheck, W. 1926/29: Kohlengeologie der österreichischen Teilstaaten, 2. - Kattowitzer Buchdruckerei - und Verlags-Sp. Akc., 484 pp. Taf. 9-20, Katowice.

Plummer, H. J. 1926: Foraminifera of the Midway Formation in Texas. - University of Texas Bulletin no. 2644, 206 pp., pls. 1-15, Austin.

Popescu, G. 1975: Etude des Foraminifères du Miocène inférieur et moyen du nord-ouest de la Transylvanie. - Mém. Institut de Géologie. et Geophysique 23, 1-121, pls. 1-106, Bucarest.

Premru, U. 1983: Tolmač za list Ljubljana, Osnovna geološka karta SFRJ 1:100000 (Geology of the Sheet Ljubljana, Geological Map of SFRJ 1:100000). - Zvezni geološki zavod, 75 pp., Beograd (in Slovenian with English abstract).

Reiser, H. 1987: Die Foraminiferen der bayerischen Oligozän-Molasse. Systematik, Stratigraphie und Paläobathymetrie. - Zitteliana, v. 16, 3-131, pls. 1-19, München. 
Reuss, A. E. 1850: Neue Foraminiferen aus den Schichten des österreichischen Tertiärbeckens. - Denckschriften k. Akad. Wiss. Mathem.-naturwiss. Cl., 1, 365-390, pls. 1-6, Wien.

Reuss, A. E. 1851: Ueber die fossilen Foraminiferen und Entomostraceen der Septarienthone der Umgebung von Berlin. - Zeitschrift Deutsch. Geol. Ges., 3, 49-92, pls. 3-7, Berlin.

Reuss, A. E. 1863: Beiträge zur Kenntniss der tertiären Foraminiferen-Fauna. - Sitzungsber. K. Akad. Wiss. math.-naturw. Cl., 48, 36-71, pls. 18, Wien.

Reuss, A. E. 1866: Die Foraminiferen, Anthozoen und Bryozoen des deutschen Septarienthones. Ein Beitrag zur Fauna der mitteloligocänen Tertiärschichten. - Denkschriften K. Akad. Wiss. math.-naturw. Cl. 25, 117-214, pls. 1-4, Wien

Rijavec, L. 1958: Stratigraphische Bemerkungen über die Bohrung Brnica 6 (Synklinale von Tüffer, Slovenien). - Verh. Geol. Bundesanst., 1-3, 55-58, Wien.

Rolle, 1857: Geologische Untersuchungen in der Gegend zwischen Weitenstein, WindischGratz, Cilli und Oberburg in Unter-Steiermark. Jahrb. k.k. Geol. Reichsanst., 8/3, 403-465, Wien.

Rolle, F. 1858: Über die geologische Stellung der Sotzka-Schichten. - Sitzungsberichte k. Akad. Wiss., mathem.-naturwiss. Cl., 30, 3-33, Wien.

Royden, L.H. \& Baldi, T. 1988: Early Cenozoic Tectonics and Paleogeography of the Pannonian and Surrounding Regions. In: L.H. Royden \& F. Horváth (eds.), The Pannonian Basin: A Study In basin Evolution. - The American Association of Petroleum Geologists, The Hungarian Geological Society, AAPG Memoir 45, 1-16. Oklahoma, Budapest.

Ruiz de Gaona, M. \& Colom, G. 1950 , Estudios sobre las sinecias de los foraminiferos eocénicos de la vertiente meridional del Pirineo (Cataluna-Vizcaya). - Estudios Geologicos, 6 (12), 293-434, Madrid.

Sacal, V. \& Debourle, A. 1957: Foraminifères deuitaine, $2^{\mathrm{e}}$ partie. Peneroplidae à Victoriellidae. - Mém. Soc. géol. France (nouvelle série), 78, 1-88, pls. 1-78, Paris.

Schweighauser, J. 1951: Ein Vorkommen von Neoalveolina aus dem vicentinischen Obereocaen. - Eclogae geol. Helv. 44, 465-469, Basel.

Serra-Kiel, J., Hottinger, L., Caus, E., Drobne, K., Ferrandez, C., Jauhri, A., Less, G., K., Pignatti, J., Pavlovec, R., Samso, J. M., Schaub, H., Sirel, Strougo, A., E., Tambareau, Y., Tosquella, J. \& Zakrevskaya, E. 1998: Larger foraminiferal biostratigraphy of the Tethyan Paleocene and Eocene. - Bull. Soc. géol. France, 169, no 2, 281299, Paris.

Setiawan, J. R. 1983: Foraminifera and microfacies of the type Priabonian. - Micropal. Bull, 29, 1-173, Utrecht.

Sirel, E. 1976: Description of the new species Eoannularia conica n. sp. and new obserations on the Upper Lutetian - Lower Priabonian limestone in the Darende-Gürün (west of Malatya) region. Bull. Geol. Soc.Turkey, 19, 79-82, pls. 1-2, Ankara.

Stur, D. 1864: Bemerkungen über die Geologie von Unter-Steiermark. - Jahrb. k.k. Geol. Reichsanst., 14/3, 439-444, Wien.
Stur, 1871: Geologie der Steiermark. Geognostisch-montanistischer Ver. Steiermark, 654 pp., Graz.

Szöts, E. 1956: La limite entre le Paléogène et le Néogène et le problème des étages Chattien et Aquitanien. - Acta geol. Hung., 4/2, 209-219, Budapest.

Sztràko s, K. 1982: Les Foraminifères de la marne de Buda et la limite Éocène-Oligocène en Hongrie. - Cahiers de Micropaléontologie 4, 1-48, pls. 1-39, Paris.

Sztràko s, K. 1987a: Les Foraminifères bartoniens et priaboniens des couches a "Tritaxia szaboi" de Hongrie et essai de reconstitution paléogéographique de la Montagne Centrale de Hongrie au Bartonien et Priabonien. - Cahiers de Micropaléontologie 2 (1), 5-37, pls. 1-23, Paris.

Sztràkos, K. 1987b: Le genre Pavonitina (Foraminifère) dans le Cénozoïk européen. - Revue de Micropaléontologie, 30 (2), 128-138, Paris.

Sikić, L., Simunić, A., Pikija, M. \& Šimunić, A., 1976: Gornji eocen na području Ravne gore (sjeverozapadna Hrvatska). - Geološki vjesnik, 29, 191-197, Zagreb.

Teller, F. 1889: Zur Kenntniss der Tertiärablagerungendes Gebietes von Neuhaus bei Cilli in Südsteiermark. - Verh. k. k. Geol. Reichsant., 12, 234-246, Wien.

Teller, F. 1896: Erläuterungen zur Geologischen Karte der östlichen Ausläufer der Karnischen und Julischen Alpen (Ostkarawanken und Steiner Alpen) 1.75000. - K. k. Geologische Reichsanst., 262 pp., Wien.

Teller, F. 1898: Erläuterungen zur Geologischen Karte der Oesterr.-ungar. Monarchie, Prassberg a. d. Sann 1.75000. - K. k. Geologische Reichsanst,167 pp., Wien.

Teller, F. 1907: Geologische Karte Cilli-Ratschach 1. 75000. - K. k. Geologische Reichsanst, Wien.

Terquem, O. 1878: Les Foraminifères et les Entomostracés-Ostracodes du Pliocène Supérieur de l'île de Rhode. - Mém. Soc. Géol. France sér. 3, 1, 1-135, pls. 1-9, Paris

Thenius, E. 1959: Tertiär, 2. In: Fr. Lotze, Handbuch der Stratigraphischen Geologie, 3/2. Ferdinand Enke Verlag, 328 pp., Stuttgart.

Uhlig, V. 1886: Ueber eine Microfauna aus dem Alttertiär der westgalizischen Karpathen. Jahrbuch. Geol. Reichsanstalt, 36, 141-214, pls. 26 , Wien

Wenger, W. F. 1987: Die Foraminiferen des Miozäns der Bayerischen Molasse und ihre stratigraphische sowie paläogeographische Auswertung. - Zitteliana, 16, 173-340, pls. 1-22, München.

Witt Puyt, J. F. C. de 1941: Geologische und paläontologische Beschreibung der Umgebung von Ljubuški, Hercegovina, 99 pp., pls. 1-5, Utrecht. Zollik ofer, Th. 1859: Die geologischen Verhältnisse von Untersteiermark. Gegend südlich der Sann und Wolska. - Jahrb. k. k. Geol. Reichsanst., 10/2, 157-219, 1 Taf., Wien.

Zollikofer, 1861/62: Die geologischen Verhältnisse des südöstichen Theiles von UnterSteiermark. - Jahrb. k. k. Geol. Reichsanst., 12/3, 311-367, 1 Taf., Wien. 